\title{
COMPUTING CLOSED ESSENTIAL SURFACES IN KNOT COMPLEMENTS
}

\author{
BENJAMIN A. BURTON, ALEXANDER COWARD, AND STEPHAN TILLMANN
}

\begin{abstract}
We present a new, practical algorithm to test whether a knot complement contains a closed essential surface. This property has important theoretical and algorithmic consequences; however, systematically testing it has until now been infeasibly slow, and current techniques only apply to specific families of knots. As a testament to its practicality, we run the algorithm over a comprehensive body of 2979 knots, including the two 20 -crossing dodecahedral knots, yielding results that were not previously known.

The algorithm derives from the original Jaco-Oertel framework, involves both enumeration and optimisation procedures, and combines several techniques from normal surface theory. This represents substantial progress in the practical implementation of normal surface theory, in that we can systematically solve a theoretically double exponential-time problem for significant inputs. Our methods are relevant for other difficult computational problems in 3-manifold theory, ranging from testing for Haken-ness to the recognition problem for knots, links and 3-manifolds.
\end{abstract}

\section{INTRODUCTION}

In the study of 3-manifolds, essential surfaces have been of central importance since Haken's seminal work in the 1960s. An essential surface may be regarded as 'topologically minimal', and there has since been extensive research into 3-manifolds, called Haken 3-manifolds, that contain an essential surface. The existence of such a surface has profound consequences for both the topology and geometry of a 3-manifold [13, 18, 19, 20, 26, 29].

Given any closed 3-manifold, specified by a triangulation, it is a theorem of Jaco and Oertel [15] from 1984 that one may algorithmically test for the existence of a closed essential surface. However, their algorithm has significant intricacies and is of doubleexponential complexity in terms of the input size, putting it well beyond the scope of a practical implementation.

In this paper we present for the first time a practical algorithm that can systematically test a significant class of 3-manifolds for the existence of a closed essential surface, and which is both efficient in practice and always conclusive. To illustrate its power, we run this algorithm over a comprehensive body of input data, yielding computer proofs of new mathematical results.

The 3-manifolds we examine in this paper are the motivating spaces for 3-manifold theory: knot complements. These are the spaces that arise by removing a knotted curve

The authors are supported by the Australian Research Council under the Discovery Projects funding scheme (projects DP1094516 and DP110101104). 
from 3-dimensional space, although our methods can be extended to apply to a far wider class of 3-manifolds. See the full version of this paper for generalisations. In this paper we work with two collections of input data. First, for each of the 2977 non-trivial prime knots that can be drawn with a diagram of at most 12 crossings, we determine whether its complement contains a closed essential surface. If there is no such surface, the knot is called small, otherwise we call it large. Second, we apply our algorithm to resolve, in the affirmative, a question of Michel Boileau [4] who enquired whether two special 20-crossing knots called dodecahedral knots contain a closed essential surface in their complements. This question was recently also independently resolved by Jessica Banks [3] using non-computational techniques.

The algorithm presented here is theoretically significant because it is the first algorithm in the literature for testing largeness of arbitrary knots. However, more important is its practical significance: this is the first conclusive algorithm of this type that is implemented and fast enough for real-world use. The prior state-of-the-art algorithm for detecting essential surfaces was used to prove that the Weber-Seifert dodecahedral space is Haken [10]; however, although it resolved a long-standing open problem, this prior algorithm relies on heuristic methods that only work for certain triangulations, and are only conclusive if no essential surface exists. In contrast, the algorithm described here can work with arbitrary triangulations of knot complements, and is found to be effective regardless of the final result.

Our methods can be applied to related invariants of knots and 3-manifolds. For instance, the smallest genus $g$ of a closed essential surface is an important knot invariant about which little is known for the case $g \geq 2$, and our algorithm opens the door to formulating and testing new hypotheses. These methods may also be extended to test a wide variety of 3-manifolds for Haken-ness and related properties. More broadly, iterated exponential complexity algorithms arise frequently in 3-manifold theory, and our methods give an outline for how such problems, like the recognition problem for knots and 3-manifolds, may one day be within the realm of a practical implementation.

We base our work on the framework of the Jaco-Oertel algorithm for testing for closed incompressible surfaces. This uses normal surfaces, which allow us to translate topological questions about surfaces into the setting of integer and linear programming. The framework consists of two stages: the first constructs a finite list of candidate essential surfaces, and the second tests each surface in the list to see if it is essential. A key difficulty with this framework, which our algorithm also inherits, is that both stages have running times that are worst-case exponential in their respective input sizes, and combining them in any obvious way leads to a double-exponential complexity solution.

Despite this significant hurdle, we introduce several innovations that cut down the running time enormously for both stages. Our optimisation for the first stage involves a combination of established techniques that, though well understood individually, require new ideas and theory in order to work harmoniously together. For the second stage we combine branch-and-bound techniques from integer programming with the Jaco-Rubinstein procedure for crushing surfaces within triangulations. In more detail: 
- For the first stage (enumerating candidate essential surfaces), we combine several techniques. First, we wish to create a triangulation for each knot complement that contains as few tetrahedra as possible. If one uses classical triangulations one needs as many as 50 tetrahedra for some knots in the 12-crossing tables, a size for which enumerating candidate surfaces is thoroughly infeasible even for modern high-performance machines. We therefore use ideal triangulations for knot complements, which are decompositions of these spaces into tetrahedra with their vertices removed. These introduce some significant theoretical difficulties, but they are much smaller with roughly half as many tetrahedra.

Second, we use a variant of normal surface theory based on quadrilateral coordinates. The appeal is that this brings the dimension of the underlying integer and linear programming problems down from $7 t$ in the classical setting to $3 t$, where $t$ is the number tetrahedra in the input 3-manifold. These coordinates were known to Thurston and Jaco in the 1980s, and first appeared in print in work of Tollefson 28 .

A theoretical difficulty arises when combining ideal triangulations with quadrilateral coordinates: this introduces objects called spun-normal surfaces, which are properly embedded non-compact surfaces (essentially built from infinitely many pieces). We counter this by introducing extra linear constraints called boundary equations which, with the development of appropriate theory, restrict the solution space in question to closed surfaces only. In particular, using an extension of the work of Jaco and Oertel [15] from compact manifolds to noncompact manifolds by Kang [21], we show in Theorem 2 that for each manifold under consideration, there is a finite, constructible set of normal surfaces with the property that if the manifold in question contains a closed essential surface, then one must exist in this set.

- For the second stage (testing whether a candidate surface is essential), the JacoOertel approach cuts along each candidate surface and inspects the boundary of the resulting 3-manifold to see if it admits a compression disc (such a disc certifies that a surface is non-essential). The key difficulty is that one requires a new triangulation for the cut-open 3-manifold: since the candidate surface may be very complicated, any natural scheme for cutting and re-triangulating yields a new triangulation with exponentially many tetrahedra in the worst case, taking us far beyond the realm in which normal surface theory has traditionally been feasible in practice. Since these new triangulations are the input for stage two, which is itself exponential time, we now see where the double exponential arises, and why the Jaco-Oertel framework has long been considered far from practical.

We resolve this significant problem using a blend of techniques. First, we use strong simplification heuristics to reduce the number of tetrahedra. Next, we replace the traditional (and very expensive) enumeration-based search for compression discs with an optimisation process that maximises Euler characteristic. This uses the branch-and-bound techniques of [9], and allows us to 
quickly focus on a single candidate compression disc. We employ the crushing techniques of Jaco and Rubinstein [16] to quickly test whether this is indeed a compression disc, and (crucially) to reduce the size of the triangulation if it is not.

More generally, this issue of iterated-exponential complexity, coming from cutting and re-triangulating, arises with ubiquity when considering objects called normal hierarchies. These hierarchies are key when solving more difficult problems such as the recognition problem for knots and 3-manifolds. Our approach to stage two is both fast in practice and theoretically correct, making it a substantial breakthrough that indicates that a practical implementation of these more difficult algorithms might indeed be possible.

\section{PRELiminaries}

2.1. Knots, surfaces and triangulations. A 3-manifold is a mathematical object that locally looks like 3-dimensional Euclidean space. Because every topological 3manifold admits precisely one piecewise-linear structure (up to PL-homeomorphism) [23], in practice this means that 3-manifolds may be studied via triangulations. A triangulation of a compact 3-manifold $M$ is a description of $M$ as the disjoint union of a finite collection of 3 -simplices with their faces identified in pairs, as shown in Figure 1 .

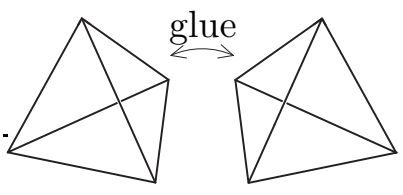

FiguRE 1. A 3-manifold may be specified by a triangulation.

A triangulation $\mathcal{T}$ for a 3-manifold $M$ gives rise to vertices, edges, faces and tetrahedra in $M$. Edges whose interior lies in the interior of $M$ are called interior edges, and edges that lie entirely on the boundary of $M$ are called boundary edges. In practice, a tetrahedron in $M$ might not be embedded; for instance, we even allow two faces of a tetrahedron to be identified in $M$. For a precise description of our set-up, please see A.3, and for an example, see Appendix B.

Such a triangulation can only specify a compact 3-manifold. If instead of identifying the faces of tetrahedra, we identify the faces of a finite collection of tetrahedra minus their vertices, this constitutes an ideal triangulation for the resulting non-compact quotient space.

The 3-manifolds we study in this paper are knot complements. These are 3-manifolds obtained by removing a knot, which is knotted closed curve in $\mathbb{R}^{3}$, from 3-dimensional Euclidean space. In practice it is convenient to compactify $\mathbb{R}^{3}$ with a point at infinity, yielding a compact 3-manifold called the 3-sphere, denoted $S^{3}$. One then removes the knot from $S^{3}$ instead. For a knot $K$ we call the resulting non-compact 3-manifold $S^{3} \backslash K$ the complement of $K$. Knot complements always have ideal triangulations [27, Proposition 1.2]. 
If instead we remove from $S^{3}$ a small open neighborhood of a knot $K$ we obtain a compact 3-manifold called the exterior of $K$. Since they are compact, knot exteriors may be specified by triangulations. There are well established techniques for translating between an ideal triangulation for a knot complement and a triangulation for the corresponding knot exterior.

A knot $K$ is called non-trivial if it is not the boundary of an embedded disc in $S^{3}$.

In this paper we are interested in closed essential surfaces in knot complements. We define these now. Let $K$ be a knot, and let $M$ be the complement of $K$. A connected two-sided closed surface with positive genus $S$, embedded in $M$, is a closed essential surface in $M$ if the following properties hold: (i) the surface $S$ is incompressible (as defined below); and (ii) the surface $S$ is not boundary parallel, that is, not ambient isotopic to a small tube running around $K$.

The definition of incompressible is as follows. A compression disc for an embedded surface $S$ in a 3-manifold $M$ is an embedded disc $D \subset M$ for which (i) $D \cap S$ equals the boundary of $D$ (denoted $\partial D$ ); and (ii) $\partial D$ is a non-trivial curve in $S$ (meaning $\partial D$ does not bound a disc in $S$ ). If the surface $S$ admits a compression disc, then we say $S$ is compressible, otherwise $S$ is incompressible. An equivalent, algebraic criterion can be found in $\$$ A.2.

2.2. Quadrilateral coordinates and $Q-$ matching equations. We use normal surface theory to search for essential surfaces. A normal surface in a (possibly ideal) triangulation $\mathcal{T}$ is a properly embedded surface which intersects each tetrahedron of $\mathcal{T}$ in a disjoint collection of triangles and quadrilaterals, as shown in Figure 2. These triangles and quadrilaterals are called normal discs. In an ideal triangulation of a noncompact 3-manifold, a normal surface may contain infinitely many triangles; such a surface is called spun-normal.
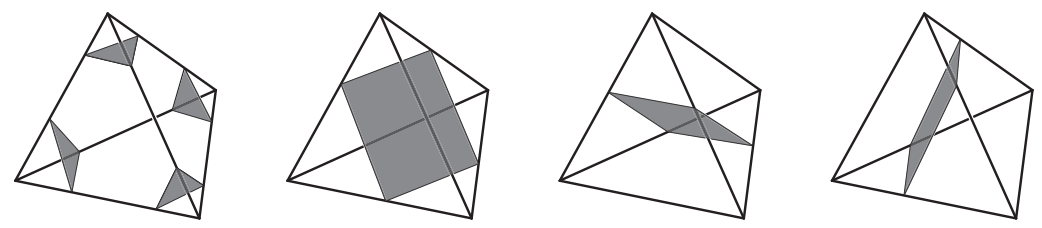

FiguRE 2. The seven types of normal disc in a tetrahedron.

We now describe an algebraic approach to normal surfaces. The key observation is that each normal surface contains finitely many quadrilateral discs, and is uniquely determined (up to normal isotopy) by these quadrilateral discs. Here a normal isotopy of $M$ is an isotopy that keeps all simplices of all dimensions fixed. Let $\square$ denote the set of all normal isotopy classes, or types, of normal quadrilateral discs, so that $|\square|=3 t$ where $t$ is the number of tetrahedra in $\mathcal{T}$. We identify $\mathbb{R}^{\square}$ with $\mathbb{R}^{3 t}$. Given a normal surface $S$, let $x(S) \in \mathbb{R}^{\square}=\mathbb{R}^{3 t}$ denote the integer vector for which each coordinate $x(S)(q)$ counts the number of quadrilateral discs in $S$ of type $q \in \square$. This normal $Q$-coordinate $x(S)$ satisfies the following two algebraic conditions.

First, $x(S)$ is admissible. A vector $x \in \mathbb{R}^{\square}$ is admissible if $x \geq 0$, and for each tetrahedron $x$ is non-zero on at most one of its three quadrilateral types. This reflects 
the fact that an embedded surface cannot contain two different types of quadrilateral in the same tetrahedron.

Second, $x(S)$ satisfies a linear equation for each interior edge in $M$, termed a $Q_{-}$ matching equation. Intuitively, these equations arise from the fact that as one circumnavigates the earth, one crosses the equator from north to south as often as one crosses it from south to north. We now give the precise form of these equations. To simplify the discussion, we assume that $M$ is oriented and all tetrahedra are given the induced orientation; see [27, Section 2.9] for details.

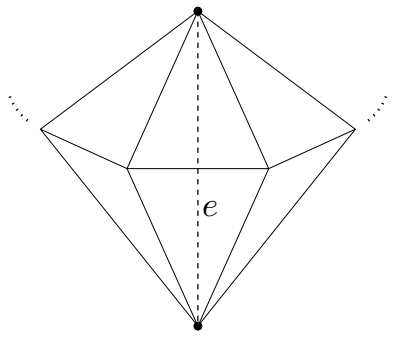

(a) The abstract neighbourhood $B(e)$

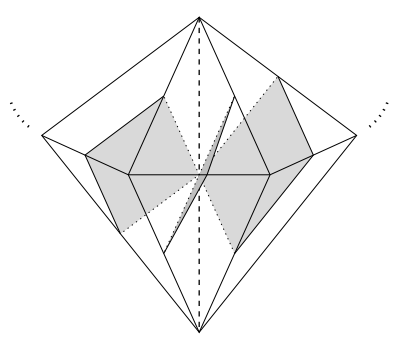

(b) Positive slope

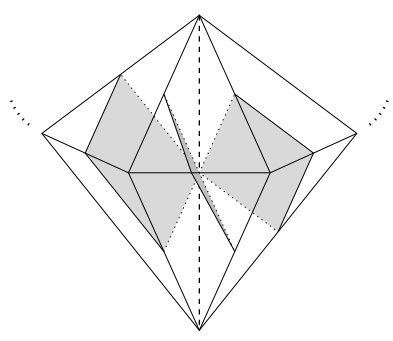

(c) Negative slope

FiguRE 3. Slopes of quadrilaterals

Consider the collection $\mathcal{C}$ of all (ideal) tetrahedra meeting at an edge $e$ in $M$ (including $k$ copies of tetrahedron $\sigma$ if $e$ occurs $k$ times as an edge in $\sigma$ ). We form the abstract neighbourhood $B(e)$ of $e$ by pairwise identifying faces of tetrahedra in $\mathcal{C}$ such that there is a well defined quotient map from $B(e)$ to the neighbourhood of $e$ in $M$; see Figure 3(a) for an illustration. Then $B(e)$ is a ball (possibly with finitely many points missing on its boundary). We think of the (ideal) endpoints of $e$ as the poles of its boundary sphere, and the remaining points as positioned on the equator.

Let $\sigma$ be a tetrahedron in $\mathcal{C}$. The boundary square of a normal quadrilateral of type $q$ in $\sigma$ meets the equator of $\partial B(e)$ if and only it has a vertex on $e$. In this case, it has a slope of a well-defined sign on $\partial B(e)$ which is independent of the orientation of $e$. Refer to Figures 3(b) and 3(c), which show quadrilaterals with positive and negative slopes respectively.

Given a quadrilateral type $q$ and an edge $e$, there is a total weight wt $_{e}(q)$ of $q$ at $e$, which records the sum of all slopes of $q$ at $e$ (we sum because $q$ might meet $e$ more than once, if $e$ appears as multiple edges of the same tetrahedron). If $q$ has no corner on $e$, then we set $\mathrm{wt}_{e}(q)=0$. Given edge $e$ in $M$, the $Q$-matching equation of $e$ is then defined by $0=\sum_{q \in \square} \operatorname{wt}_{e}(q) x(q)$.

Theorem 1. For each $x \in \mathbb{R}^{\square}$ with the properties that $x$ has integral coordinates, $x$ is admissible and $x$ satisfies the $Q$-matching equations, there is a (possibly non-compact) normal surface $S$ such that $x=x(S)$. Moreover, $S$ is unique up to normal isotopy and adding or removing vertex linking surfaces, i.e. normal surfaces consisting entirely of normal triangles.

For a proof of Theorem 11 see Theorem 2.1 of [21] or Theorem 2.4 of [27]. 
The set of all $x \in \mathbb{R}^{\square}$ with the property that (i) $x \geq 0$ and (ii) $x$ satisfies the $Q$-matching equations is denoted $Q(\mathcal{T})$. This naturally is a polyhedral cone. Note however that the set of all admissible $x \in \mathbb{R}^{\square}$ typically meets $Q(\mathcal{T})$ in a non-convex set.

2.3. Crushing triangulations. The crushing process of Jaco and Rubinstein [16] plays an important role in our algorithms, and we informally outline this process here. For the formal details we refer the reader to [16].

Let $S$ be a two-sided normal surface in a triangulation $\mathcal{T}$ of a compact orientable 3manifold $M$. To crush $S$ in $\mathcal{T}$, we (i) cut $\mathcal{T}$ open along $S$, which splits each tetrahedron into a number of (typically non-tetrahedral) pieces; (ii) crush each resulting copy of $S$ on the boundary to a point, which converts these pieces into tetrahedra, footballs and/or pillows as shown in Figure 4(a); (iii) flatten each football or pillow to an edge or triangle respectively, as shown in Figure 4(b).
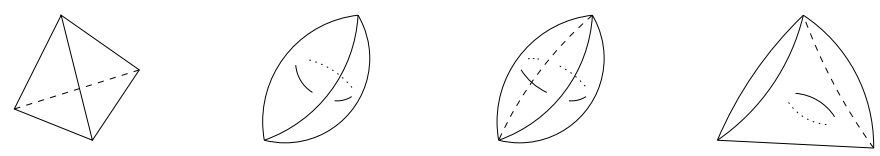

(a) Pieces after crushing $S$ to a point
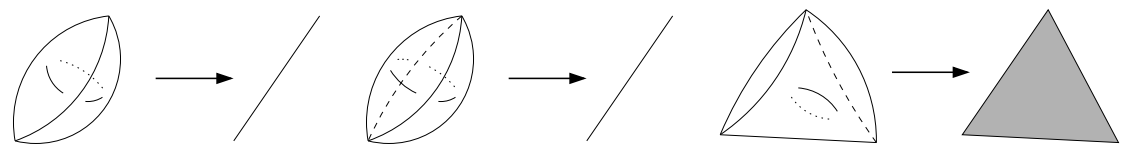

(b) Flattening footballs and pillows

Figure 4. Steps in the Jaco-Rubinstein crushing process

The result is a new collection of tetrahedra with a new set of face identifications. We emphasise that we only keep track of face identifications between tetrahedra: any "pinched" edges or vertices fall apart, and any lower-dimensional components with no tetrahedra at all simply disappear. The resulting structure might not represent a 3-manifold triangulation, and even if it does the flattening operations might have changed the underlying 3-manifold in ways that we did not intend.

Although crushing can cause a myriad of problems in general, Jaco and Rubinstein show that in some cases the operation behaves extremely well [16]. In particular, if $S$ is a normal sphere or disc, then after crushing we always obtain a triangulation of some 3-manifold $M^{\prime}$ (possibly disconnected, and possibly empty) that is obtained from the original $M$ by zero or more of the following operations:

- cutting manifolds open along spheres and filling the resulting boundary spheres with 3-balls;

- cutting manifolds open along properly embedded discs;

- capping boundary spheres of manifolds with 3-balls;

- deleting entire connected components that are any of the 3-ball, the 3-sphere, projective space $\mathbb{R} P^{3}$, the lens space $L(3,1)$ or the product space $S^{2} \times S^{1}$. 
An important observation is that the number of tetrahedra that remain after crushing is precisely the number of tetrahedra that do not contain quadrilaterals of $S$.

\section{Closed normal surfaces in $Q-$ SPaCE}

In this section we introduce the linear boundary equations, with which we restrict the normal surface solution space to closed surfaces only.

Let our knot complement be $M=S^{3} \backslash K$. The ideal triangulation $\mathcal{T}$ of $M$ has one ideal vertex, and its link is a torus. We view this torus $T$ as made up of normal triangles, one near each corner of each ideal tetrahedron. Let $\gamma \in H_{1}(T ; \mathbb{R})$. We now describe an associated linear functional $\nu(\gamma): \mathbb{R}^{\square} \rightarrow \mathbb{R}$, which measures the behaviour along $\gamma$ of a normal surface near the ideal vertex. The idea is similar to the intuitive description of the $Q$-matching equations. As one goes along $\gamma$ and looks down into the manifold, normal quadrilaterals will (as Jeff Weeks puts it) come up from below or drop down out of sight. If the total number coming up minus the total number dropping down is non-zero, then the surface spirals towards the knot in the cross section $\gamma \times[0, \infty) \subset T \times[0, \infty)$ and the sign indicates the direction, see Figure $6(\mathrm{~b})$. If this number is zero, then after a suitable isotopy the surface meets the cross section in a (possibly empty or infinite) union of circles, see Figure 6(c).

The torus $T$ has an induced triangulation consisting of normal triangles. Represent $\gamma$ by an oriented path on $T$, which is disjoint from the 0 -skeleton and meets the 1skeleton transversely. Each edge of a triangle in $T$ is a normal arc. Give the edges of each triangle in $T$ transverse orientations pointing into the triangle and labelled by the quadrilateral types sharing the normal arc with the triangle; see Figure 5. We then define $\nu(\gamma)$ as follows. Choosing any starting point on $\gamma$, we read off a formal linear combination of quadrilateral types $q$ by taking $+q$ each time the corresponding edge is crossed with the transverse orientation, and $-q$ each time it is crossed against the transverse orientation (where each edge in $T$ is counted twice - using the two adjacent triangles).
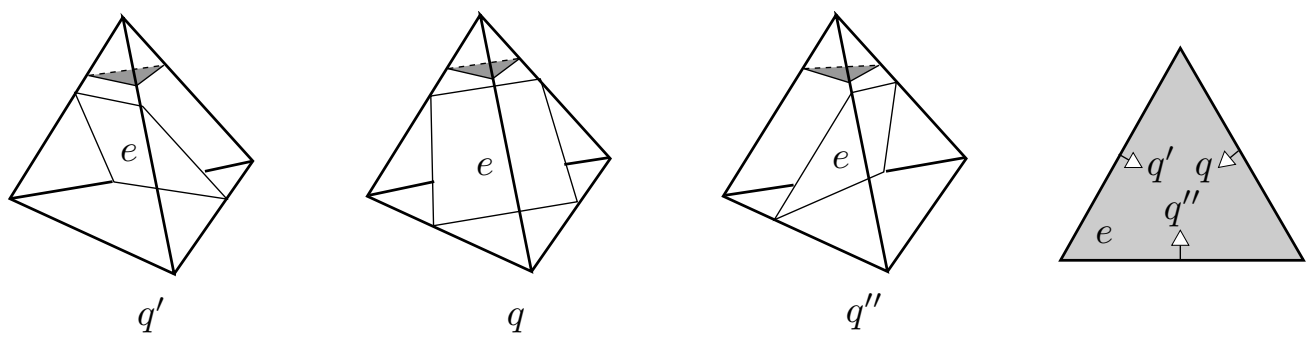

Figure 5. Coming up and dropping down

Evaluating $\nu(\gamma)$ at some $x \in \mathbb{R}^{\square}$ gives a real number $\nu_{x}(\gamma)$. For example, taking a small loop around a vertex in $T$ and setting this equal to zero gives the $Q$-matching equation of the corresponding edge in $M$; see Figure 6(a). For each $x \in Q(\mathcal{T})$, the resulting map $\nu_{x}: H_{1}(T ; \mathbb{R}) \rightarrow \mathbb{R}$ is a well-defined homomorphism, which has the property that the surface in Theorem 1 is closed if and only if $\nu_{x}=0$ (see [27], 
Proposition 3.3). Since $\nu_{x}: H_{1}(T ; \mathbb{R}) \rightarrow \mathbb{R}$ is a homomorphism, it is trivial if and only if we have $\nu_{x}(\alpha)=0=\nu_{x}(\beta)$ for any basis $\{\alpha, \beta\}$ of $H_{1}(T ; \mathbb{R})$.

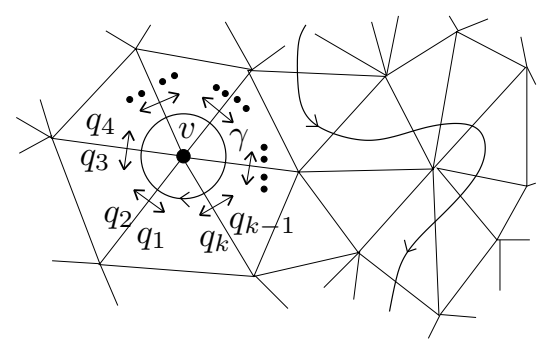

(a) $0=\nu_{x}(\gamma)=\sum_{i=1}^{k}(-1)^{i} x\left(q_{i}\right)$ is the $Q$-matching equation

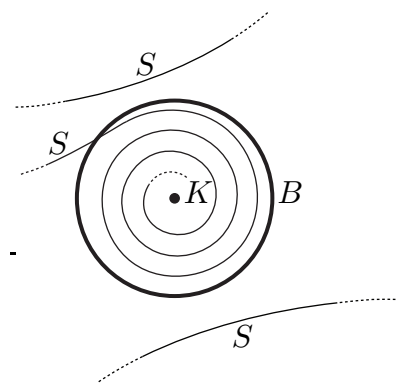

(b) spun $\Longleftrightarrow \nu_{x} \neq 0$

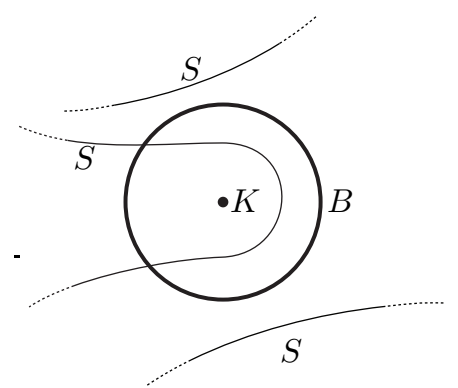

(c) not spun $\Longleftrightarrow \nu_{x}=0$

Figure 6. Boundary map determines $Q-$ matching equations and spinning

We define $Q_{0}(\mathcal{T})=Q(\mathcal{T}) \cap\left\{x \mid \nu_{x}=0\right\}$, and call a two-sided, connected normal surface $F$ with $x(F)$ on an extremal ray of $Q_{0}(\mathcal{T})$ a $Q_{0}$-vertex surface. The following result is based on the seminal work of Jaco and Oertel [15]:

Theorem 2. Suppose $M$ is the complement of a non-trivial knot in $S^{3}$. If $M$ contains a closed essential surface $S$, then there is a normal, closed essential surface $F$ with the property that $x(F)$ lies on an extremal ray of $Q_{0}(\mathcal{T})$. Moreover, if $\chi(S)<0$, then there is such $F$ with $\chi(F)<0$.

Sketch of proof. A complete proof of a more general statement is given in Appendix C. The key ideas are as follows. Given a closed essential surface in $M$, a standard argument shows that there is a normal closed essential surface in $M$. Amongst all normal surfaces isotopic (but not necessarily normally isotopic) to this, choose one that has minimal number of intersections with the 1-skeleton (this is the PL analogue of a minimal surface). Denote this surface $S$.

If $S$ is not a vertex surface, one can write it using a so-called Haken sum of vertex surfaces, which is a geometric realisation of the sum of $Q$-coordinate vectors. However, a complication arises, since only a multiple of $S$ is a Haken sum of vertex surfaces, and only up to vertex linking tori; that is, $n S+\Sigma=\sum n_{i} V_{i}=V+W$, where $V$ is a vertex surface, $\Sigma$ is vertex linking, and all other terms are combined into the surface $W$. Building on Jaco and Oertel [15], Kang [21, Theorem 5.4] shows that $V$ and $W$ are incompressible. Since Euler characteristic is additive under Haken sum, the result follows if $\chi(S)<0$. If $\chi(S)=0$, additional work is required to show that an essential torus cannot be written as a Haken sum of boundary parallel tori.

\section{Algorithms}

Here we describe the new algorithm to test whether a knot is large or small (i.e., whether its complement contains a closed essential surface). In this extended abstract we restrict our attention to the common setting of knots in the 3 -sphere $S^{3}$. See the full 
version of this paper for an extension to the more general setting of links in arbitrary closed orientable 3-manifolds, as well as searching for essential surfaces in arbitrary closed orientable 3-manifolds (without knots or links).

We present the algorithm in two stages below. Algorithm 3 describes a subroutine to test whether a given closed surface is incompressible. Algorithm 5 is the main algorithm: it uses the results of Section 3 to identify candidate essential surfaces, and runs Algorithm 3 over each.

These algorithms contain a number of high-level and often intricate procedures, many of which are described in separate papers. For each algorithm, we discuss these procedures in further detail after presenting the overall algorithm structure.

Algorithm 3 (Testing for incompressibility). Let $\mathcal{T}$ be an ideal triangulation of a non-compact 3-manifold $M$ that is the complement of a non-trivial knot in $S^{3}$. Let $S$ be a closed two-sided normal surface of genus $g \geq 1$ within $\mathcal{T}$. To test whether $S$ is incompressible in $M$ :

(1) Truncate the ideal vertex of $\mathcal{T}$ to obtain a compact manifold with boundary, cut $\mathcal{T}$ open along the surface $S$, and retriangulate. The result is a pair of triangulations $\mathcal{T}_{1}, \mathcal{T}_{2}$ representing two compact manifolds with boundary $M_{1}, M_{2}$ (one on each side of $S$ in $M$ ).

Let $B_{1}, B_{2}$ be the genus $g$ boundary components of $\mathcal{T}_{1}$ and $\mathcal{T}_{2}$ respectively that correspond to the surface $S$. Without loss of generality, suppose that the truncated ideal vertex was on the side of $M_{2}$; therefore $\mathcal{T}_{2}$ has an additional boundary torus, which we denote $B_{v}$.

(2) For each $i=1,2$ :

(a) Simplify $\mathcal{T}_{i}$ into a triangulation with no internal vertices and only one vertex on each boundary component, without increasing the number of tetrahedra. Let the resulting number of tetrahedra in $\mathcal{T}_{i}$ be $n$.

(b) Search for a connected normal surface $E$ in $\mathcal{T}_{i}$ that is not a vertex link, has positive Euler characteristic, and (for the case $i=2$ ) does not meet the torus boundary $B_{v}$.

(c) If no such $E$ exists, then there is no compressing disc for $S$ in $M_{i}$. If $i=1$ then try $i=2$ instead, and if $i=2$ then terminate with the result that $S$ is incompressible.

(d) Otherwise, crush the surface $E$ as explained in Section 2.3 to obtain a new triangulation $\mathcal{T}_{i}^{\prime}$ (possibly disconnected, or possibly empty) with strictly fewer than $n$ tetrahedra. If some component of $\mathcal{T}_{i}^{\prime}$ has the same genus boundary (or boundaries) as $\mathcal{T}_{i}$ then it represents the same manifold $M_{i}$, and we return to step 2 a using this component of $\mathcal{T}_{i}^{\prime}$ instead. Otherwise we terminate with the result that $S$ is not incompressible.

Regarding the individual steps of this algorithm:

- Step 1 requires us to truncate an ideal vertex and cut a triangulation open along a normal surface. These are standard (though intricate) procedures. To truncate a vertex we subdivide tetrahedra and then remove the immediate 
neighbourhood of the vertex. To cut along a normal surface, see [10] for a description of a manageable implementation.

- Step 2a requires us to simplify a triangulation to use the fewest possible vertices, without increasing the number of tetrahedra. For this we begin with the rich polynomial-time simplification heuristics described in [6]. In practice, for all 2979 knots that we consider in Section 5, this is sufficient to reduce the triangulation to the desired number of vertices.

If there are still extraneous vertices, we can remove these using the crushing techniques of Jaco and Rubinstein [16, Section 5.2]. This might fail, but only if $\partial M_{i}$ has a compressing disc, or two boundary components of $M_{i}$ are separated by a sphere; both cases immediately certify that the surface $S$ is compressible, and we can terminate immediately.

- Step $2 \mathrm{~b}$ requires us to locate a connected normal surface $E$ in $\mathcal{T}_{i}$ that is not a vertex link, has positive Euler characteristic, and does not meet the torus boundary $B_{v}$. For this we use the recent method of [9, Algorithm 11], which draws on combinatorial optimisation techniques: in essence we run a sequence of linear programs over a combinatorial search tree, and prune this tree using tailored branch-and-bound methods. See [9] for details.

We note that this search is the bottleneck of Algorithm 3: the search is worst-case exponential time, though in practice it often runs much faster [9]. The exposition in [9] works in the setting where the underlying triangulation is a knot complement, but the methods work equally well in our setting here. To avoid the boundary component $B_{v}$, we simply remove all normal discs that touch $B_{v}$ from our coordinate system.

Theorem 4. Algorithm 3 terminates, and its output is correct.

Proof. The algorithm terminates because each time we loop back to step 2a we have fewer tetrahedra than before. Correctness is more interesting: there are many claims in the algorithm statement that require proof. Full proofs are given in Appendix D, the key ideas are as follows.

- In step 1 we claim that cutting along $S$ yields two (disconnected) compact manifolds. This follows from the fact that every closed surface embedded in the 3 -sphere is separating.

- In step 2c we claim that, if the surface $E$ cannot be found in $\mathcal{T}_{1}$ and it cannot be found in $\mathcal{T}_{2}$, then the original surface $S$ must be incompressible. This is because otherwise, by results of Jaco and Oertel [15], there must be a normal compressing disc on one side of $S$.

- In step 2d we make several claims. First, the new triangulation $\mathcal{T}_{i}^{\prime}$ has strictly fewer tetrahedra because $E$ is not a vertex link. Second, we claim that if $\mathcal{T}_{i}^{\prime}$ has a component with the same genus boundary (or boundaries) as $\mathcal{T}_{i}$ then it represents the same manifold $M_{i}$, and otherwise $S$ is compressible; this is because the "destructive" side-effects of the crushing process reduce the boundary genus by cutting along compressing discs for $S$. 
There are additional complications involving irreducibility; again see Appendix $\mathrm{D}$ for details.

Algorithm 5 (Testing whether a knot is large or small). Let $K$ be a non-trivial knot in $S^{3}$. To test whether $K$ is large or small:

(1) Build an ideal triangulation $\mathcal{T}$ of the complement of $K$ in $S^{3}$.

(2) Enumerate all extremal rays of $Q_{0}(\mathcal{T})$; denote these $\mathbf{e}_{1}, \ldots, \mathbf{e}_{k}$. For each extreme ray $\mathbf{e}_{i}$, let $S_{i}$ be the unique connected two-sided normal surface for which $x\left(S_{i}\right)$ lies on $\mathbf{e}_{i}$. Ignore all surfaces $S_{i}$ that are spheres.

(3) For each remaining surface $S_{i}$, use algorithm 3 to test whether $S_{i}$ is incompressible in $\mathcal{T}$. If any $S_{i}$ is incompressible and is not a torus, then terminate with the result that $K$ is large. If no $S_{i}$ is incompressible, then terminate with the result that $K$ is small.

(4) Otherwise the only incompressible surfaces in our list are tori. For each incompressible torus $S_{i}$, test whether $S_{i}$ is boundary parallel by (i) cutting $\mathcal{T}$ open along $S_{i}$, and then (ii) using the Jaco-Tollefson algorithm [17, Algorithm 9.7] to test whether one of the resulting components is the product space $($ Torus $) \times[0,1]$. If all incompressible tori are found to be boundary parallel then $K$ is small, and otherwise $K$ is large.

Regarding the individual steps:

- Step 1 requires us to triangulate the complement of $K$. Hass et al. [14] show how to build a compact triangulation (with boundary triangles). To make this an ideal triangulation we cone over the boundary, and retriangulate to remove internal (non-ideal) vertices.

- Step 2 requires us to enumerate all extremal rays of $Q_{0}(\mathcal{T})$. This is an expensive procedure (which is unavoidable, since there is a worst-case exponential number of extremal rays). For this we use the recent state-of-the-art tree traversal method [8], which is tailored to the constraints and pathologies of normal surface theory and is found to be highly effective for larger problems. The tree traversal method works in the larger cone $Q(\mathcal{T})$, but it is a simple matter to insert the two additional linear equations corresponding to $\nu_{x}=0$.

We also note that it is simple to identify the unique closed two-sided normal surface for which $Q(S)$ lies on the extremal ray e. Specifically, $Q(S)$ is either the smallest integer vector on e or, if that vector yields a one-sided surface, then its double.

- If we do not reach a conclusive result in step 3 , then step 4 requires us to run the Jaco-Tollefson algorithm to test whether any incompressible torus is boundaryparallel. This algorithm is expensive: it requires us to work in a larger normal coordinate system, solve difficult enumeration problems, and perform intricate geometric operations.

However, it is rare that we should reach this situation, and indeed for all 2979 knots that we consider in Section 5, this scenario never occurs. For some knots (e.g., satellite knots) it cannot be avoided, but there are additional fast 
methods for avoiding the Jaco-Tollefson algorithm even in these settings, which we describe in the full version of this paper.

Theorem 6. Algorithm 5 terminates, and its output is correct.

Proof. It is clear that the algorithm terminates (there is no looping), and the correctness follows immediately from Theorems 2 and 4 . For details see Appendix E.

\section{Computational Results}

Here we describe the results of running the algorithms of Section 4 over significant collections of input knots. These computational results emphasise that the new largeness testing algorithm is both feasible to implement, and fast enough to be practical for non-trivial inputs - both features that distinguish it from many of its peers in algorithmic low-dimensional topology.

The algorithms were implemented in $\mathrm{C}+\mathrm{+}$ using the software package Regina [5, 7]. The code is available from http://www.maths.uq.edu.au/ bab/code/, and works with the forthcoming Regina version 4.94. Supporting data for the computations described here, including triangulations of the knot complements and the corresponding lists of admissible extreme rays of $Q_{0}(\mathcal{T})$, can be downloaded from this same location.

All running times reported here are measured on a single $2.93 \mathrm{GHz}$ Intel Core i7 CPU.

5.1. The census of knots up to 12 crossings. Our first data set is the census of all 2977 non-trivial prime knots that can be represented with $\leq 12$ crossings. Ideal triangulations of the knot complements were extracted from the SnapPy census tables [12], and then further simplified where possible using Regina's greedy heuristics [6] to yield a final set of input triangulations ranging from 2-26 tetrahedra in size.

The algorithms ran successfully over all 2977 triangulations, yielding the following results:

Theorem 7. Of the 2977 distinct non-trivial prime knots with up to 12 crossings, 1019 are large and 1958 are small.

A full list of all 1019 large knots can be found in Appendix F. Regarding performance:

- The enumeration of extremal rays of $Q_{0}(\mathcal{T})$ was extremely fast, with a maximum time of 47 seconds, and a median time of just 0.08 seconds. This is a clear illustration of the benefits we obtain from Theorem 2, which allows us to work in the restricted cone $Q_{0}(\mathcal{T})$ instead of the (typically much larger) cone $Q(\mathcal{T})$.

The number of extremal rays of $Q_{0}(\mathcal{T})$ ranged from 0 (for the figure eight knot complement) up to 509 (for one of the 26-tetrahedron triangulations), with a median of 33 .

- Testing whether each candidate surface was essential was also extremely fast in most (but not all) cases. For each knot complement, we can sum the times required to process all candidate surfaces: the median time over all 2977 knots was $\sim 3.6$ seconds, and all but three of the knots had a processing time of under 12 minutes. 
The remaining three knots, however, were significantly slower to process. One required $\sim 3.9$ hours, one required $\sim 12.2$ hours, and one (the knot 12a $\mathrm{a}_{0779}$ ) was still running after 6 days. However, in a striking illustration of how the algorithms depend strongly upon the underlying triangulations, when the code was run with a different random seed (which affected the simplification heuristics, and hence the triangulations obtained after slicing along surfaces), this worst-case knot $12 \mathrm{a}_{0779}$ was fully processed in under 4 minutes.

5.2. The dodecahedral knots. We now turn our attention to the dodecahedral knots $D_{f}$ and $D_{s}$ as described by Aitchison and Rubinstein [1. These two knots exhibit remarkable properties [2, 24], and each can be represented with 20 crossings [2]. Running our algorithms over them yields the following results:

Theorem 8. The two dodecahedral knots $D_{f}$ and $D_{s}$ are both large. In particular, their complements contain closed essential surfaces of genus 3.

We work with ideal triangulations of the knots $D_{f}$ and $D_{s}$ with 46 and 47 tetrahedra respectively, which were kindly provided by Craig Hodgson. These are significantly larger than the knots from the 12-crossing census; indeed, triangulations of this size are typically considered well outside the range of feasibility for normal normal surface theory. Happily our algorithms now prove otherwise:

- This time the enumeration of extremal rays of $Q_{0}(\mathcal{T})$ was the bottleneck: for $D_{f}$ and $D_{s}$ this enumeration took roughly 2.8 and 2.4 days respectively. The number of admissible extremal rays was 72272 and 73609 respectively.

- To test whether candidate surfaces were essential, the knot $D_{s}$ was completely processed in under 3 minutes; in contrast, $D_{f}$ required roughly 4.4 hours. Once again, we see that this part of the algorithm depends heavily upon the underlying triangulation: when running with a different random seed, $D_{f}$ was likewise processed in just a few minutes.

\section{REFERENCES}

1. I. R. Aitchison and J. H. Rubinstein, Combinatorial cubings, cusps, and the dodecahedral knots, Topology '90 (Columbus, OH, 1990), Ohio State Univ. Math. Res. Inst. Publ., vol. 1, de Gruyter, Berlin, 1992, pp. 17-26.

2. Iain R. Aitchison and J. Hyam Rubinstein, Geodesic surfaces in knot complements, Experiment. Math. 6 (1997), no. 2, 137-150.

3. Jessica Banks, The complement of a dodecahedral knot contains an essential closed surface, http://thales.math.uqam.ca/ banksj/, 2012.

4. Michel Boileau, Private correspondence, 2012.

5. Benjamin A. Burton, Introducing Regina, the 3-manifold topology software, Experiment. Math. 13 (2004), no. 3, 267-272.

6. __ Computational topology with Regina: Algorithms, heuristics and implementations, To appear in Geometry \& Topology Down Under, Amer. Math. Soc., arXiv: 1208. 2504, 2012.

7. Benjamin A. Burton, Ryan Budney, William Pettersson, et al., Regina: Software for 3-manifold topology and normal surface theory, http://regina.sourceforge.net/, 1999-2012.

8. Benjamin A. Burton and Melih Ozlen, A tree traversal algorithm for decision problems in knot theory and 3-manifold topology, To appear in Algorithmica, arXiv:1010.6200, 2010. 
9. _ A fast branching algorithm for unknot recognition with experimental polynomial-time behaviour, Preprint, arXiv:1211.1079, November 2012.

10. Benjamin A. Burton, J. Hyam Rubinstein, and Stephan Tillmann, The Weber-Seifert dodecahedral space is non-Haken, Trans. Amer. Math. Soc. 364 (2012), no. 2, 911-932.

11. Jae Choon Cha and Charles Livingston, KnotInfo: Table of knot invariants, http://www. indiana.edu/ knotinfo, accessed November 2011.

12. Marc Culler, Nathan M. Dunfield, and Jeffrey R. Weeks, SnapPy, a computer program for studying the geometry and topology of 3-manifolds, http://snappy.computop.org/, 1991-2011.

13. Wolfgang Haken, Some results on surfaces in 3-manifolds, Studies in Modern Topology, Studies in Mathematics, no. 5, Math. Assoc. Amer., 1968, pp. 39-98.

14. Joel Hass, Jeffrey C. Lagarias, and Nicholas Pippenger, The computational complexity of knot and link problems, J. Assoc. Comput. Mach. 46 (1999), no. 2, 185-211.

15. William Jaco and Ulrich Oertel, An algorithm to decide if a 3-manifold is a Haken manifold, Topology 23 (1984), no. 2, 195-209.

16. William Jaco and J. Hyam Rubinstein, 0-efficient triangulations of 3-manifolds, J. Differential Geom. 65 (2003), no. 1, 61-168.

17. William Jaco and Jeffrey L. Tollefson, Algorithms for the complete decomposition of a closed 3-manifold, Illinois J. Math. 39 (1995), no. 3, 358-406.

18. William H. Jaco and Peter B. Shalen, Seifert fibered spaces in 3-manifolds, Mem. Amer. Math. Soc. 21 (1979), no. 220, viii+192.

19. Klaus Johannson, Homotopy equivalences of 3-manifolds with boundaries, Lecture Notes in Mathematics, vol. 761, Springer, Berlin, 1979.

20. __ On the mapping class group of simple 3-manifolds, Topology of Low-Dimensional Manifolds (Proc. Second Sussex Conf., Chelwood Gate, 1977), Lecture Notes in Math., vol. 722, Springer, Berlin, 1979, pp. 48-66.

21. Ensil Kang, Normal surfaces in non-compact 3-manifolds, J. Aust. Math. Soc. 78 (2005), no. 3, 305-321.

22. Sergei Matveev, Algorithmic topology and classification of 3-manifolds, Algorithms and Computation in Mathematics, no. 9, Springer, Berlin, 2003.

23. Edwin E. Moise, Affine structures in 3-manifolds. V. The triangulation theorem and Hauptvermutung, Ann. of Math. (2) 56 (1952), 96-114.

24. Walter D. Neumann and Alan W. Reid, Arithmetic of hyperbolic manifolds, Topology '90 (Columbus, OH, 1990), Ohio State Univ. Math. Res. Inst. Publ., vol. 1, de Gruyter, Berlin, 1992, pp. 273310.

25. Peter B. Shalen, Representations of 3-manifold groups, Handbook of Geometric Topology, NorthHolland, Amsterdam, 2002, pp. 955-1044.

26. William P. Thurston, Hyperbolic structures on 3-manifolds. I. Deformation of acylindrical manifolds, Ann. of Math. (2) 124 (1986), no. 2, 203-246.

27. Stephan Tillmann, Normal surfaces in topologically finite 3-manifolds, Enseign. Math. (2) 54 (2008), 329-380.

28. Jeffrey L. Tollefson, Normal surface Q-theory, Pacific J. Math. 183 (1998), no. 2, 359-374.

29. Friedhelm Waldhausen, On irreducible 3-manifolds which are sufficiently large, Ann. of Math. (2) 87 (1968), 56-88. 


\section{ApPEndiX: AdDitional PROOFs, EXAMPles AND DATA}

This paper is concerned with finding special surfaces in 3-manifolds. To keep the paper short, we mainly considered the case of finding a closed essential surface in a knot exterior in $S^{3}$, and have introduced the bare minimum of the new theory and algorithms required for this application. The most natural generalisation of our results and algorithms is to the setting of compact 3-manifolds with boundary consisting of a finite union of tori. These manifolds are related to the Geometrisation Theorem of Thurston and Perelman, as they are key building blocks in the so-called JSJ decomposition of a closed, irreducible, orientable 3-manifold into geometric pieces.

\section{Appendix A. General Definitions}

In this first appendix, we give more technical definitions of knot and link manifolds, essential surfaces, triangulations and normal surfaces.

A.1. Knots and 3-manifolds. Suppose $M$ is an orientable 3-manifold (possibly with boundary). There are two key properties that are often required of $M$ in geometric topology. The first is that every embedded 2-sphere in $M$ bounds a ball to at least one side; in this case $M$ is irreducible. The second is that for each boundary component $B$ of $M$, the inclusion homomorphism $\pi_{1}(B) \rightarrow \pi_{1}(M)$ is injective; in this case $M$ is $\partial$-irreducible. (A geometric interpretation of this algebraic property is given in the next section.) For instance, the 3 -sphere $S^{3}$ is irreducible, and if $K$ is an embedding of $S^{1}$ in $S^{3}$, called a knot, then the knot exterior $M=S^{3} \backslash N(K)$, where $N(K)$ is a small open neighbourhood of $K$, is also irreducible, and it is $\partial$-irreducible if and only if $K$ is non-trivial. The knot complement $S^{3} \backslash K$ is homeomorphic to the interior of the knot exterior $S^{3} \backslash N(K)$, and it is sometimes useful to switch between the complement (a non-compact manifold) and the exterior (a manifold-with-boundary).

A.2. Surfaces in 3-manifolds. The following definition of an essential surface, along with an extensive discussion of their properties, can be found in Shalen [25], §1.5.

Definition 9 (Essential surface). A properly embedded surface $S$ in the compact, irreducible, orientable 3-manifold $M$ is essential if it has the following properties:

(1) $S$ is bicollared;

(2) the inclusion homomorphism $\pi_{1}\left(S_{i}\right) \rightarrow \pi_{1}(M)$ is injective for each component $S_{i}$ of $S$

(3) no component of $S$ is a 2 -sphere;

(4) no component of $S$ is boundary parallel; and

(5) $S$ is non-empty.

Of interest to this paper is the following geometric interpretation of the second property, see Shalen [25] for more details. A compression disc for the surface $S$ is a disc $D \subset M$ such that $D \cap S=\partial D$ and $\partial D$ is homotopically non-trivial in $S$ (i.e. does not bound a disc on $S$ ). In particular, if $S$ has a compression disc, then $\pi_{1}\left(S_{i}\right) \rightarrow \pi_{1}(M)$ is not injective for some component of $S$. It follows from classical work of Papakyriakopoulos that the converse is also true. Detecting compression discs is the topic of Section 4 . 
If the surface $S$ has non-empty boundary and is properly embedded in a 3 -manifold with boundary, there is an additional requirement that one has in order for $S$ to be topologically significant; namely that it be $\partial$-incompressible. In the case of interest for this paper, where all boundary components of $M$ are tori, it turns out that incompressible implies $\partial$-incompressible. This is one reason why our algorithms most naturally generalise to the class of link complements.

A.3. Triangulations. The notation of [16] and [27] will be used in this paper. A triangulation, $\mathcal{T}$, of a compact 3-manifold $M$ consists of a union of pairwise disjoint 3-simplices, $\widetilde{\Delta}=\left\{\sigma_{1}, \ldots, \sigma_{t}\right\}$, a set of face pairings, $\Phi$, and a natural quotient map $p: \widetilde{\Delta} \rightarrow \widetilde{\Delta} / \Phi=M$. Since the quotient map is injective on the interior of each $3-$ simplex, we will refer to the image of a 3-simplex in $M$ as a tetrahedron and to its faces, edges and vertices with respect to the pre-image. Similarly for images of $2-, 1-$ and 0 -simplices, which will be referred to as faces, edges and vertices in $M$ respectively. If an edge is contained in $\partial M$, then it is termed a boundary edge; otherwise it is an interior edge.

If $M$ is the interior of a compact manifold with non-empty boundary, an ideal triangulation, $\mathcal{T}$, of $M$ consists of a union of pairwise disjoint 3-simplices, $\widetilde{\Delta}$, a set of face pairings, $\Phi$, and a natural quotient map $p: \widetilde{\Delta} \rightarrow \widetilde{\Delta} / \Phi=P$, such that $M=P \backslash P^{(0)}$ is the complement of the 0 -skeleton. The quotient space $P$ is usually called a pseudomanifold.

For brevity, we will refer to a 3 -manifold $M$ imbued with a (possibly ideal) triangulation $\mathcal{T}=(\widetilde{\Delta}, \Phi, p)$ as a triangulated 3-manifold. Throughout, we will assume that $M$ is oriented, that all tetrahedra in $M$ are oriented coherently and the tetrahedra in $\widetilde{\Delta}$ are given the induced orientation.

A.4. Normal surfaces. A normal corner is an interior point of a 1-simplex. A normal arc is a properly embedded straight line segment on a 2 -simplex with boundary consisting of normal corners. A normal disc is a properly embedded disc in a 3-simplex whose boundary consists of normal arcs no two of which are contained on the same face of the 3-simplex; moreover, the normal disc is the cone over its boundary with cone point the barycentre of its normal corners. It follows that the boundary of a normal disc consists of either three or four normal arcs, and it is accordingly called a normal triangle or a normal quadrilateral. Moreover, a normal disc is uniquely determined by its intersection with the 1 -skeleton.

A normal isotopy is an isotopy of $M$ that leaves all simplices invariant. Up to normal isotopy, there are 7 types of normal discs in each tetrahedron. We denote $\square$ the set of all isotopy classes of normal quadrilaterals in $\widetilde{\Delta}$, and identify this with the set of all isotopy classes of normal quadrilaterals in $M$ via $p$. Following Haken, we will connect topology to linear programming via certain functions $x: \square \rightarrow \mathbb{R}^{3 t}$.

A normal surface $S$ in a triangulated 3-manifold is a properly embedded surface which intersects each 3-simplex in a union of pairwise disjoint normal discs; such a surface is often termed a spun-normal surface if one of its connected components contains infinitely many normal discs. 
A.5. Standard coordinates. In classical normal surface theory one only considers normal surfaces where each tetrahedron contains at most finitely many disjoint normal disks. The number of parallel copies each type of normal disk specifies the normal surface, and in this way normal surfaces may be studied via $7 t$-dimensional vectors with positive integer coordinates, where $t$ is the number of tetrahedra in the triangulation under consideration. These surfaces can be described by a set of linear equations arising from the fact that the normal discs in one tetrahedron have to match up with normal discs in each adjacent tetrahedron across their common face. If $\Delta$ denotes the set of all normal isotopy classes of normal triangles, then the natural coordinate projection $\mathbb{R}^{\Delta \cup \square} \rightarrow \mathbb{R}^{\square}$ takes the standard coordinate of a normal surface to its $Q$-coordinate.

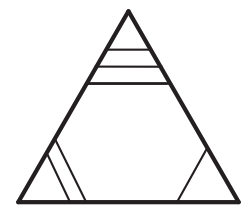

FIGURE 7. The intersection of a normal surface with a typical face of the 2-skeleton.

Consider a face of the 2-skeleton of the triangulation of $M$. Any normal surface in $M$ must intersect this triangle in normal arcs, that is arcs which start and end on different edges. There are three types of normal arc in any face of the 2-skeleton, as shown in Figure 7. Each of these represents an edge of a normal disc on each side. These normal discs must match up, as shown in Figure 8. Note that on each side of a triangle of the 2-skeleton, there are only two possible types of normal disc, one triangle and one quadrilateral, which can give rise to each type of normal arc.

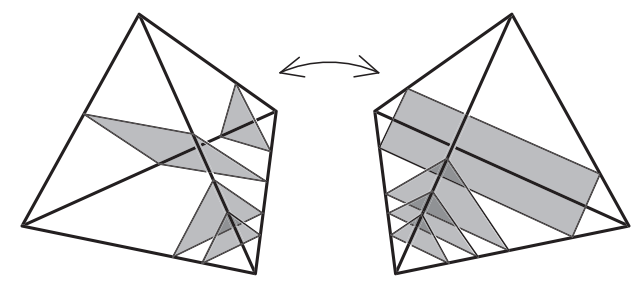

Figure 8. The normal discs must match up on the common face of two adjacent tetrahedra.

Let $t_{1}, q_{1}$ be the normal triangle and quadrilateral types in one tetrahedron, sharing the same normal arc type with the normal triangle and quadrilateral types $t_{2}$ and $q_{2}$ in an adjacent tetrahedron. We then obtain the linear equation

$$
t_{1}+q_{1}=t_{2}+q_{2}
$$

with three such equations for each internal face of the triangulation. The space of all non-negative solutions to these equations in $\mathbb{R}^{\Delta \cup \square}$ is the usual normal surface cone. 


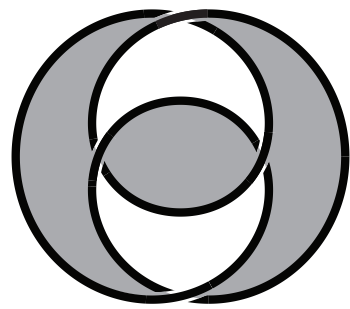

(a) The figure 8 knot spans a Klein bottle
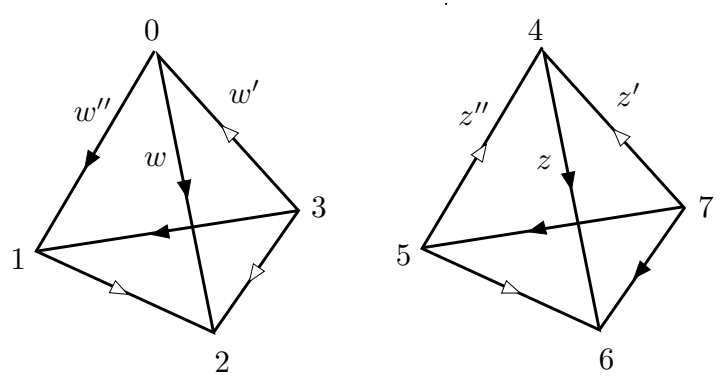

(b) Thurston's ideal triangulation of the complement

Figure 9. The figure eight knot

\section{Appendix B. Example: The figure-eight knot}

Let $M$ denote the complement of the figure eight knot, which is shown in Figure 9(a). An oriented, ideal triangulation of $M$ is encoded in Figure 9(b). Since $M$ is oriented, we may compute the $Q$-matching equations from the figure. To simplify notation, we use the dual labelling scheme of [27, and denote the quadrilateral types dual to the edges labelled $w^{(k)}$ and $z^{(k)}$ by $p^{(k)}$ and $q^{(k)}$ respectively. The $Q$-matching equations for the two edges are equivalent, and one has:

$$
0=p+p^{\prime}-2 p^{\prime \prime}+q+q^{\prime}-2 q^{\prime \prime} .
$$

This implies that the cone $Q(\mathcal{T})$ is five-dimensional. A direct calculation reveals that $Q(\mathcal{T})$ has four admissible extremal rays; all have minimal representative a oncepunctured Klein bottle; such a Klein bottle is shown in Figure 9(a). Their normal $Q$-coordinates and boundary slopes are listed in Table1. This calculation in particular shows that no spun-normal surface is a Seifert surface for the knot.

\begin{tabular}{c|r|r|r} 
solution & $\nu(\mu)$ & $\nu(\lambda)$ & slope \\
\hline$(2,0,0,0,0,1)$ & 1 & 4 & -4 \\
$(0,2,0,0,0,1)$ & -1 & 4 & 4 \\
$(0,0,1,2,0,0)$ & -1 & -4 & -4 \\
$(0,0,1,0,2,0)$ & 1 & -4 & 4
\end{tabular}

TABLE 1. Normal surface in the figure eight knot complement

The induced triangulation of a vertex linking torus, $T$, is shown in Figure 10. The dual labelling allows us to read off the linear functional $\nu(\gamma)$ for the path $\gamma$ that is transverse to the 1-skeleton on $T$. If the path $\gamma$ on $T$ exits a triangle across the edge opposite the vertex with label $w^{(k)}$, then in $\nu(\gamma)$, we record $-p^{(k)}$, and if it enters a triangle across the edge opposite the vertex labelled $w^{(k)}$, then in $\nu(\gamma)$, we record $+p^{(k)}$. Similarly for the labels $z^{(k)}$. 


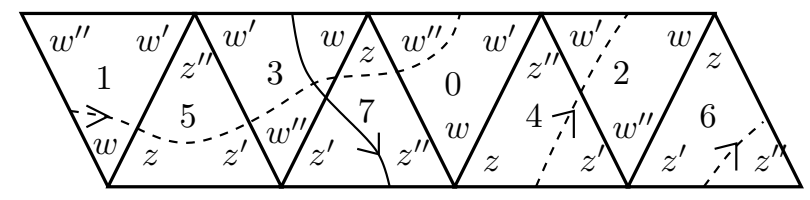

Figure 10. The induced triangulation of the vertex linking torus, where the sides of the rectangle are identified by translations parallel to its sides and triangle $i$ is dual to vertex $i$ in Figure 9. The shown elementary curves are the standard meridian $\mu$ (solid) and longitude $\lambda$ (dashed).

In this fashion (and using the $Q$-matching equation to simplify the expressions), we determine the linear functionals associated to the standard peripheral curves:

$$
\begin{aligned}
& \nu(\lambda)=2 p+2 p^{\prime}-4 p^{\prime \prime}, \\
& \nu(\mu)=-p^{\prime}+p^{\prime \prime}-q+q^{\prime \prime} .
\end{aligned}
$$

One can now verify that $Q_{0}(\mathcal{T})=\{0\}$. Whence any closed embedded normal surface is a union of vertex linking tori.

\section{Appendix C. Proof of Theorem 2}

In this appendix, we prove Theorem 2 in the more general setting of a 3-manifold with boundary consisting of a union of tori. The definition of the boundary map and of $Q_{0}(\mathcal{T})$ is generalised to the case of multiple boundary components as follows. For each vertex linking torus $T_{k}$, we obtain a well-defined homomorphism $\nu_{k, x}: H_{1}\left(T_{k} ; \mathbb{R}\right) \rightarrow \mathbb{R}$, and we define $\nu_{x}=\oplus_{k} \nu_{k, x}$, where the sum is taken over all ideal vertices. The surface in Theorem 1 is closed if and only if $\nu_{x}=0$ (see [27], Proposition 3.3). We then define $Q_{0}(\mathcal{T})=Q(\mathcal{T}) \cap\left\{x \mid \nu_{x}=0\right\}$, and call a 2-sided, connected normal, surface $F$ with $Q(F)$ on an extremal ray of $Q_{0}(\mathcal{T})$ a $Q_{0}$-vertex surface.

The weight of the normal surface $F$ is the cardinality of its intersection with the 1 -skeleton, $\operatorname{wt}(F)=\left|F \cap M^{(1)}\right|$. If $F$ is closed, then its weight is finite.

Two normal surfaces are compatible if they do not meet a tetrahedron in quadrilateral discs of different types. In this case, the sum of their normal coordinates is the coordinate of a normal surface. Suppose $F_{1}$ and $F_{2}$ are normal surfaces that are compatible, not vertex linking surfaces, and in general position. Then $x\left(F_{1}\right)+x\left(F_{2}\right)$ is an admissible solution to the $Q$-matching equations, and hence represented by a unique normal surface without vertex linking components; denote this surface $F$. The surface $F$ is obtained geometrically as follows. At each component of $F_{1} \cap F_{2}$, there is a natural choice of regular switch between normal discs, such that the result is again a normal surface. See Figure 11 for some possible configurations involving only two discs. Denote $N\left(F_{1} \cap F_{2}\right)$ a small, open, tubular neighbourhood of $F_{1} \cap F_{2}$. The connected components of $\left(F_{1} \cup F_{2}\right) \backslash N\left(F_{1} \cap F_{2}\right)$ are termed patches.

Deleting any vertex linking tori that arise gives the surface $F$, and we write $F+\Sigma=$ $F_{1}+F_{2}$, where $\Sigma$ is (possibly empty or a possibly infinite) union of vertex linking tori. This is called the Haken sum of $F_{1}$ and $F_{2}$. Both weight and Euler characteristic are 


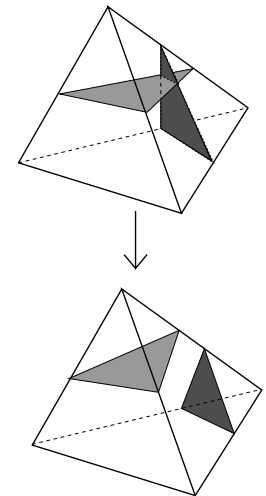

(a)

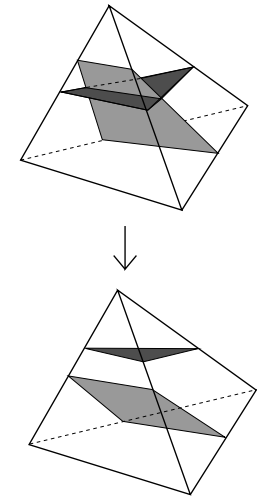

(b)

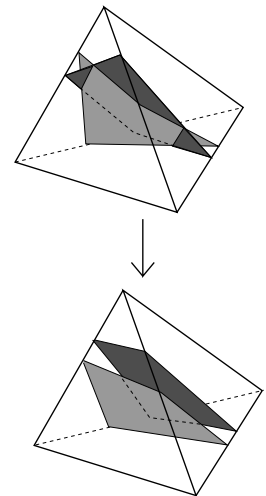

(c)

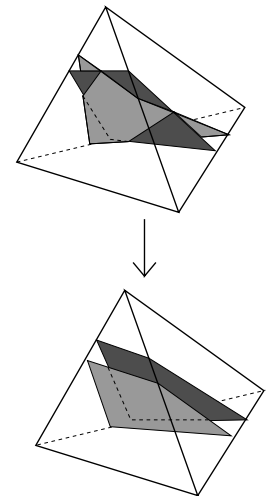

(d)

FiguRE 11. Regular exchange of normal discs

additive under this sum. So we have

$$
\begin{aligned}
\mathrm{wt}\left(F_{1}\right)+\mathrm{wt}\left(F_{2}\right) & =\mathrm{wt}(F)+\mathrm{wt}(\Sigma), \\
\chi\left(F_{1}\right)+\chi\left(F_{2}\right) & =\chi(F),
\end{aligned}
$$

since $\chi(\Sigma)=0$.

The sum $F+\Sigma=F_{1}+F_{2}$ is said to be in reduced form if there is no Haken sum $F+\Sigma^{\prime}=F_{1}^{\prime}+F_{2}^{\prime}$, where $F_{i}^{\prime}$ is isotopic to $F_{i}$ in $M, F_{1}^{\prime} \cap F_{2}^{\prime}$ has fewer components than $F_{1} \cap F_{2}$ and $\Sigma^{\prime}$ is a union of vertex linking tori. It should be noted that in these two sums, the embedding of $F$ in $M$ is the same (these are not equalities up to isotopy), and that any sum can be changed to a sum in reduced form.

Theorem 2. Suppose $M$ is the interior of a compact, irreducible and $\partial$-irreducible manifold with boundary consisting of a union of tori, and let $\mathcal{T}$ be an ideal triangulation of $M$. If $M$ contains a closed, essential surface $S$, then there is a normal, closed essential surface $F$ with the property that $x(F)$ lies on an extremal ray of $Q_{0}(\mathcal{T})$. Moreover, if $\chi(S)<0$, then there is such $F$ with $\chi(F)<0$.

Proof. Suppose $M$ contains a closed, essential surface. It follows from a standard argument (see, for instance, [15] and [16]) that there is a closed, essential, normal surface $S$ in $M$. It remains to show that $S$ may be chosen such that $S$ is a $Q_{0}$-vertex surface. Replace $S$ by a normal surface that has least weight amongst all normal surfaces isotopic (but not necessarily normally isotopic) to $S$. Denote this least weight surface $S$ again.

Suppose $S$ is not a $Q_{0}$-vertex surface. Then

$$
n x(S)=\sum n_{i} x\left(V_{i}\right)
$$

where $n, n_{i} \in \mathbb{N}$ and either $V_{i}$ or $2 V_{i}$ is a $Q_{0}$-vertex surface for each $i$. The two cases arise from the fact that we require a $Q_{0}$-vertex surface to be 2 -sided and connected: If $V$ corresponds to the first integer lattice point on an admissible extremal ray of $Q_{0}(\mathcal{T})$ 
and $V$ is 1 -sided, then the corresponding $Q_{0}$-vertex surface is $2 V$, obtained by taking the boundary of a regular neighbourhood of $V$.

We denote $n S$ the normal surface obtained by taking $n$ parallel copies of $S$. Clearly, $x(n S)=n x(S)$, and since $S$ has least weight normal surface in its isotopy class, so does $n S$ because $S$ is 2 -sided. To sum up, $n S$ is a closed, essential, normal surface which has least weight amongst all normal surfaces in its isotopy class.

For any $i$, either $V_{i}$ or $2 V_{i}$ is a $Q_{0}$-vertex surface. In the first case, we can write

$$
n S+\Sigma=V+W,
$$

where $V=V_{i}$ and $x(W)=-x\left(V_{i}\right)+\sum n_{i} x\left(V_{i}\right)$. Now $n S$ is 2-sided and of least weight, so Kang [21], Theorem 5.4 (which is an adaptation of the proof of [15], Theorem 2.2, to this context), shows that if one writes $n S+\Sigma=V^{\prime}+W^{\prime}$ in reduced form, then $V^{\prime}$ is incompressible. Now $V^{\prime}$ is isotopic in $M$ to $V$, and hence $V$ is also incompressible. If $2 V_{i}$ is a $Q_{0}$-vertex surface, then we apply the above argument to $2 n S$, writing $2 n S+\Sigma=$ $V+W$, where $x(V)=2 x\left(V_{i}\right)$ and $x(W)=-x(V)+2 \sum n_{i} x\left(V_{i}\right)$. In either case, we obtain an incompressible $Q_{0}-$ vertex surface $V$. Since Euler characteristic is additive and $S$ is not a sphere, there is some $V_{i}$ with $\chi\left(V_{i}\right) \leq 0$. If $\chi\left(V_{i}\right)<0$, then $V$ is essential, and if $\chi\left(V_{i}\right)=0$, then $V$ may be essential or boundary-parallel. Hence if $\chi(S)<0$, the proof of the theorem is complete.

Hence assume $\chi(S)=0$, and finish the proof with an argument from the proof of Proposition 6.3.21 from [22]. For the sake of a contradiction, suppose that some $V_{i}$ is a boundary parallel torus that is not vertex linking. Write $n S+\Sigma=V+W$, where $V$ is a boundary parallel torus that is not vertex linking, and suppose that the sum is in reduced form. As in [15], Lemma 2.1, it follows that each patch is incompressible and not a disc. Denote $M_{2}$ a component of $M \backslash V$ that is homeomorphic to $V \times(0,1)$. If $W \cap M_{2} \neq \emptyset$, then it consists of a pairwise disjoint union of annuli. Choosing an innermost annulus $A \subseteq W \cap M_{2}$, there is an annulus $A^{\prime} \subseteq V$ such that $\partial A^{\prime}=\partial A$, and there is an isotopy from $A$ to $A^{\prime}$ keeping the boundaries fixed. But this implies that $V+W$ is not in reduced form, giving a contradiction.

Remark 10. The statement of Theorem 2 considerably strengthens the statement of Theorem 5.5 in [21], and our proof fills a gap in its proof.

\section{Appendix D. Proof of Theorem 4}

Here we give a full proof of correctness for Algorithm 3. Recall that this algorithm takes a closed two-sided normal surface $S$ of genus $g$ within an ideal triangulation $\mathcal{T}$ of a knot complement in $S^{3}$, and tests whether this surface is incompressible.

Theorem 4. Algorithm 3 terminates, and its output is correct.

Proof. As noted in the main body of the paper, termination is straightforward: each time we loop back to step 2a we have strictly fewer tetrahedra than before. We now devote ourselves to proving the many claims that are made throughout the statement of Algorithm 3 .

Before proceeding, however, we make a brief note regarding irreducibility. Since the underlying knot is embedded in $S^{3}$, every sphere in the complement $M$ must bound a 
ball; that is, $M$ is irreducible. As a result, the two manifolds $M_{1}$ and $M_{2}$ are likewise irreducible, with the following possible exception: it might be the case that $M_{2}$ has an embedded sphere that separates the genus $g$ boundary $B_{2}$ on one side from the torus boundary $B_{v}$ on the other. However, in this case (by reattaching $M_{1}$ ) we find that $S$ is contained in a 3 -ball within the complement $M$; in particular, $S$ has a compressing disc within $M_{1}$.

We proceed now with proofs of the various claims made in Algorithm 3 .

- In step 1 we claim that cutting along $S$ yields two compact manifolds.

This is because $S$ is a closed surface embedded in a knot complement, which itself is a submanifold of the 3-sphere. Since every closed surface in the 3-sphere is separating, the claim follows.

- In step 2c we claim that, if the surface $E$ cannot be found in $\mathcal{T}_{1}$ and it cannot be found in $\mathcal{T}_{2}$, then the original surface $S$ must be incompressible.

Suppose that $S$ were compressible, with a compression disc in some $M_{i}$. If this $M_{i}$ is irreducible, then by a result of Jaco and Oertel [15, Lemma 4.1] there is a normal compressing disc in $\mathcal{T}_{i}$. Since the underlying knot is non-trivial, this compressing disc must meet the genus $g$ boundary $B_{i}$ (not the torus boundary $B_{v}$ ), and so it is a surface of the type we are searching for. If $M_{i}$ is reducible then (from earlier) we have $i=2$, there is a compressing disc within the irreducible manifold $M_{1}$, and by the argument above the surface $E$ can be found within $\mathcal{T}_{1}$.

- In step 2d we claim that the new triangulation $\mathcal{T}_{i}^{\prime}$ has strictly fewer tetrahedra than $\mathcal{T}_{i}$.

This is because $E$ is connected but not a vertex link, and therefore contains at least one normal quadrilateral. As noted in Section 2.3, this means that at least one tetrahedron of $\mathcal{T}_{i}$ is deleted in the Jaco-Rubinstein crushing process.

- In step 2d we claim that if $\mathcal{T}_{i}^{\prime}$ has a component with the same genus boundary (or boundaries) as $\mathcal{T}_{i}$ then this component represents the same manifold $M_{i}$, and if not then $S$ is compressible.

Since the surface $E$ that we crush is connected with positive Euler characteristic and can be embedded within a knot complement (and hence the 3-sphere), it follows that $E$ is either a sphere or a disc. From Section 2.3, this means that when we crush $E$ in the triangulation $\mathcal{T}_{i}$, the resulting manifold is obtained from $M_{i}$ by zero or more of the following operations:

- undoing connected sums;

- cutting open along properly embedded discs;

- filling boundary spheres with 3-balls;

- deleting 3-ball, 3-sphere, $\mathbb{R} P^{3}, L_{3,1}$ or $S^{2} \times S^{1}$ components.

We first note that, since all of the manifolds we consider can be expressed as submanifolds of $S^{3}$, we will never create or delete an $\mathbb{R} P^{3}, L_{3,1}$ or $S^{2} \times S^{1}$ component.

Suppose that $M_{i}$ is irreducible. Then undoing a connected sum simply has the effect of creating an extra 3-sphere component. If we ever cut along a properly embedded disc that is not a compressing disc, then likewise this just 
creates an extra 3-ball component. If we cut along a compressing disc, then this yields one or two pieces with strictly smaller total boundary genus than before; moreover, since the underlying knot is non-trivial, the first such compression must take place along the genus $g$ boundary $B_{g}$ (not $B_{v}$ ) and so $S$ must be compressible. Together these observations establish the full claim above.

If $M_{i}$ is not irreducible, then as noted above $i=2$, and $M_{2}$ must contain a sphere that separates the boundary component $B_{2}$ from $B_{v}$. Here there is an extra complication: either undoing a connected sum or cutting along a non-compressing disc might have the effect of splitting the manifold into two components, one with the single boundary $B_{2}$ and the other with the single boundary $B_{v}$. In this case there will no longer be a component in $\mathcal{T}_{2}^{\prime}$ with the same genus boundaries as $\mathcal{T}_{2}$; however, as noted earlier $S$ is compressible on the side of $M_{1}$, and so the claim above remains correct.

\section{Appendix E. Proof of Theorem 6}

Our final proof is of the correctness of Algorithm 5, the full algorithm for testing whether a non-trivial knot $K$ in $S^{3}$ is large.

Theorem 6. Algorithm 5 terminates, and its output is correct.

Proof. As noted in the main body of the paper, it is clear that the algorithm terminates since there is no looping. All that remains is to prove that its output is correct.

Throughout this proof we implicitly use Theorem 4 to verify that all calls to Algorithm 3 are themselves correct. We note that, since the knot $K$ is non-trivial, the complement is irreducible and $\partial$-irreducible and so the conditions of Theorem 2 apply. We also note that all closed surfaces embedded in a knot complement in $S^{3}$ must be orientable, and so we implicitly treat all closed surfaces as orientable from here onwards.

From Theorem 2, the knot $K$ is large if and only if one of the closed normal surfaces $S_{i}$ in our list (excluding spheres) is essential. We note that each genus $\geq 2$ surface in the list is essential if and only if it is incompressible (since such a surface cannot be boundary parallel), and each torus in the list is essential if and only if it is (i) incompressible and (ii) not boundary parallel.

Steps 3 and 4 of Algorithm 5 test precisely these conditions, and so the algorithm is correct. The only reason for the complex logic in these steps is so that we can use Algorithm 3 exclusively if possible, and only fall through to the more expensive Jaco-Tollefson algorithm when absolutely necessary.

\section{Appendix F. TABles of large knots}

We finish with the detailed results of running Algorithm 5 over all 2977 knots in the census of non-trivial prime knots with $\leq 12$ crossings. The following list presents all 1019 knots in this census that are large. The knots are identified using their names in the KnotInfo database [11. 


\begin{tabular}{|c|c|c|c|c|c|c|c|c|}
\hline $8_{16}$ & $11 a_{26}$ & $11 a_{251}$ & $11 n_{21}$ & $12 a_{0006}$ & $12 a_{0089}$ & $12 a_{0185}$ & $12 a_{0348}$ & $12 a_{0509}$ \\
\hline $8_{17}$ & $11 a_{27}$ & $11 a_{252}$ & $11 n_{22}$ & $12 a_{0007}$ & $12 a_{0090}$ & $12 a_{0186}$ & $12 a_{0350}$ & $12 a_{0523}$ \\
\hline $9_{29}$ & $11 a_{28}$ & $11 a_{253}$ & $11 n_{23}$ & $12 a_{0008}$ & $12 a_{0091}$ & $12 a_{0187}$ & $12 a_{0351}$ & $12 a_{0526}$ \\
\hline $9_{32}$ & $11 a_{29}$ & $11 a_{254}$ & $11 n_{24}$ & $12 a_{0010}$ & $12 a_{0092}$ & $12 a_{0188}$ & $12 a_{0353}$ & $12 a_{0527}$ \\
\hline $9_{33}$ & $11 a_{30}$ & $11 a_{261}$ & $11 n_{25}$ & $12 a_{0011}$ & $12 a_{0093}$ & $12 a_{0189}$ & $12 a_{0355}$ & $12 a_{0554}$ \\
\hline $9_{38}$ & $11 a_{38}$ & $11 a_{262}$ & $11 n_{26}$ & $12 a_{0013}$ & $12 a_{0094}$ & $12 a_{0190}$ & $12 a_{0361}$ & $12 a_{0556}$ \\
\hline $10_{79}$ & $11 a_{43}$ & $11 a_{263}$ & $11 n_{27}$ & $12 a_{0014}$ & $12 a_{0100}$ & $12 a_{0191}$ & $12 a_{0365}$ & $12 a_{0567}$ \\
\hline $10_{80}$ & $11 a_{44}$ & $11 a_{264}$ & $11 n_{31}$ & $12 a_{0015}$ & $12 a_{0101}$ & $12 a_{0192}$ & $12 a_{0366}$ & $12 a_{0587}$ \\
\hline $10_{81}$ & $11 a_{47}$ & $11 a_{265}$ & $11 n_{32}$ & $12 a_{0023}$ & $12 a_{0102}$ & $12 a_{0195}$ & $12 a_{0377}$ & $12 a_{0588}$ \\
\hline $10_{82}$ & $11 a_{49}$ & $11 a_{266}$ & $11 n_{33}$ & $12 a_{0029}$ & $12 a_{0103}$ & $12 a_{0198}$ & $12 a_{0389}$ & $12 a_{0589}$ \\
\hline $10_{83}$ & $11 a_{52}$ & $11 a_{267}$ & $11 n_{34}$ & $12 a_{0030}$ & $12 a_{0105}$ & $12 a_{0199}$ & $12 a_{0390}$ & $12 a_{0590}$ \\
\hline $10_{84}$ & $11 a_{53}$ & $11 a_{269}$ & $11 n_{35}$ & $12 a_{0033}$ & $12 a_{0107}$ & $12 a_{0200}$ & $12 a_{0396}$ & $12 a_{0598}$ \\
\hline $10_{85}$ & $11 a_{54}$ & $11 a_{273}$ & $11 n_{36}$ & $12 a_{0036}$ & $12 a_{0108}$ & $12 a_{0201}$ & $12 a_{0398}$ & $12 a_{0599}$ \\
\hline $10_{86}$ & $11 a_{57}$ & $11 a_{274}$ & $11 n_{37}$ & $12 a_{0039}$ & $12 a_{0109}$ & $12 a_{0207}$ & $12 a_{0399}$ & $12 a_{0605}$ \\
\hline $10_{87}$ & $11 a_{66}$ & $11 a_{275}$ & $11 n_{39}$ & $12 a_{0040}$ & $12 a_{0111}$ & $12 a_{0211}$ & $12 a_{0407}$ & $12 a_{0606}$ \\
\hline $10_{88}$ & $11 a_{67}$ & $11 a_{281}$ & $11 n_{40}$ & $12 a_{0041}$ & $12 a_{0113}$ & $12 a_{0213}$ & $12 a_{0409}$ & $12 a_{0612}$ \\
\hline $10_{89}$ & $11 a_{68}$ & $11 a_{284}$ & $11 n_{41}$ & $12 a_{0043}$ & $12 a_{0114}$ & $12 a_{0214}$ & $12 a_{0410}$ & $12 a_{0613}$ \\
\hline $10_{90}$ & $11 a_{69}$ & $11 a_{286}$ & $11 n_{42}$ & $12 a_{0044}$ & $12 a_{0115}$ & $12 a_{0224}$ & $12 a_{0411}$ & $12 a_{0617}$ \\
\hline $10_{91}$ & $11 a_{70}$ & $11 a_{287}$ & $11 n_{43}$ & $12 a_{0045}$ & $12 a_{0116}$ & $12 a_{0227}$ & $12 a_{0412}$ & $12 a_{0620}$ \\
\hline $10_{92}$ & $11 a_{71}$ & $11 a_{288}$ & $11 n_{44}$ & $12 a_{0046}$ & $12 a_{0117}$ & $12 a_{0228}$ & $12 a_{0414}$ & $12 a_{0623}$ \\
\hline $10_{93}$ & $11 a_{72}$ & $11 a_{290}$ & $11 n_{45}$ & $12 a_{0047}$ & $12 a_{0119}$ & $12 a_{0231}$ & $12 a_{0416}$ & $12 a_{0624}$ \\
\hline $10_{94}$ & $11 a_{76}$ & $11 a_{291}$ & $11 n_{46}$ & $12 a_{0048}$ & $12 a_{0120}$ & $12 a_{0233}$ & $12 a_{0417}$ & $12 a_{0627}$ \\
\hline $10_{95}$ & $11 a_{79}$ & $11 a_{292}$ & $11 n_{47}$ & $12 a_{0049}$ & $12 a_{0122}$ & $12 a_{0244}$ & $12 a_{0427}$ & $12 a_{0629}$ \\
\hline $10_{96}$ & $11 a_{80}$ & $11 a_{293}$ & $11 n_{65}$ & $12 a_{0050}$ & $12 a_{0123}$ & $12 a_{0245}$ & $12 a_{0428}$ & $12 a_{0630}$ \\
\hline $10_{97}$ & $11 a_{81}$ & $11 a_{294}$ & $11 n_{66}$ & $12 a_{0051}$ & $12 a_{0125}$ & $12 a_{0260}$ & $12 a_{0429}$ & $12 a_{0633}$ \\
\hline $10_{98}$ & $11 a_{102}$ & $11 a_{298}$ & $11 n_{67}$ & $12 a_{0052}$ & $12 a_{0126}$ & $12 a_{0266}$ & $12 a_{0430}$ & $12 a_{0634}$ \\
\hline $10_{99}$ & $11 a_{123}$ & $11 a_{299}$ & $11 n_{68}$ & $12 a_{0053}$ & $12 a_{0127}$ & $12 a_{0268}$ & $12 a_{0432}$ & $12 a_{0637}$ \\
\hline $10_{109}$ & $11 a_{124}$ & $11 a_{300}$ & $11 n_{69}$ & $12 a_{0054}$ & $12 a_{0129}$ & $12 a_{0269}$ & $12 a_{0434}$ & $12 a_{0638}$ \\
\hline $10_{111}$ & $11 a_{126}$ & $11 a_{301}$ & $11 n_{71}$ & $12 a_{0055}$ & $12 a_{0131}$ & $12 a_{0271}$ & $12 a_{0435}$ & $12 a_{0639}$ \\
\hline $10_{116}$ & $11 a_{127}$ & $11 a_{304}$ & $11 n_{72}$ & $12 a_{0057}$ & $12 a_{0132}$ & $12 a_{0273}$ & $12 a_{0439}$ & $12 a_{0640}$ \\
\hline $10_{117}$ & $11 a_{129}$ & $11 a_{305}$ & $11 n_{73}$ & $12 a_{0058}$ & $12 a_{0133}$ & $12 a_{0276}$ & $12 a_{0440}$ & $12 a_{0645}$ \\
\hline $10_{122}$ & $11 a_{130}$ & $11 a_{314}$ & $11 n_{74}$ & $12 a_{0059}$ & $12 a_{0134}$ & $12 a_{0282}$ & $12 a_{0441}$ & $12 a_{0647}$ \\
\hline $10_{123}$ & $11 a_{131}$ & $11 a_{315}$ & $11 n_{75}$ & $12 a_{0060}$ & $12 a_{0135}$ & $12 a_{0283}$ & $12 a_{0446}$ & $12 a_{0654}$ \\
\hline $10_{148}$ & $11 a_{132}$ & $11 a_{316}$ & $11 n_{76}$ & $12 a_{0061}$ & $12 a_{0136}$ & $12 a_{0286}$ & $12 a_{0452}$ & $12 a_{0655}$ \\
\hline $10_{149}$ & $11 a_{138}$ & $11 a_{323}$ & $11 n_{77}$ & $12 a_{0063}$ & $12 a_{0137}$ & $12 a_{0288}$ & $12 a_{0455}$ & $12 a_{0657}$ \\
\hline $10_{150}$ & $11 a_{141}$ & $11 a_{327}$ & $11 n_{78}$ & $12 a_{0064}$ & $12 a_{0138}$ & $12 a_{0290}$ & $12 a_{0456}$ & $12 a_{0658}$ \\
\hline $10_{151}$ & $11 a_{147}$ & $11 a_{328}$ & $11 n_{80}$ & $12 a_{0065}$ & $12 a_{0139}$ & $12 a_{0291}$ & $12 a_{0460}$ & $12 a_{0659}$ \\
\hline $10_{152}$ & $11 a_{149}$ & $11 a_{332}$ & $11 n_{81}$ & $12 a_{0066}$ & $12 a_{0140}$ & $12 a_{0311}$ & $12 a_{0462}$ & $12 a_{0667}$ \\
\hline $10_{153}$ & $11 a_{150}$ & $11 a_{346}$ & $11 n_{97}$ & $12 a_{0067}$ & $12 a_{0150}$ & $12 a_{0312}$ & $12 a_{0465}$ & $12 a_{0668}$ \\
\hline $10_{154}$ & $11 a_{151}$ & $11 a_{347}$ & $11 n_{98}$ & $12 a_{0068}$ & $12 a_{0154}$ & $12 a_{0313}$ & $12 a_{0466}$ & $12 a_{0672}$ \\
\hline $11 a_{2}$ & $11 a_{152}$ & $11 a_{350}$ & $11 n_{99}$ & $12 a_{0069}$ & $12 a_{0155}$ & $12 a_{0315}$ & $12 a_{0468}$ & $12 a_{0675}$ \\
\hline $11 a_{3}$ & $11 a_{156}$ & $11 a_{353}$ & $11 n_{151}$ & $12 a_{0070}$ & $12 a_{0156}$ & $12 a_{0318}$ & $12 a_{0475}$ & $12 a_{0676}$ \\
\hline $11 a_{14}$ & $11 a_{157}$ & $11 a_{354}$ & $11 n_{152}$ & $12 a_{0071}$ & $12 a_{0157}$ & $12 a_{0328}$ & $12 a_{0479}$ & $12 a_{0680}$ \\
\hline $11 a_{15}$ & $11 a_{164}$ & $11 n_{4}$ & $11 n_{156}$ & $12 a_{0072}$ & $12 a_{0162}$ & $12 a_{0331}$ & $12 a_{0483}$ & $12 a_{0685}$ \\
\hline $11 a_{17}$ & $11 a_{167}$ & $11 n_{5}$ & $11 n_{160}$ & $12 a_{0073}$ & $12 a_{0163}$ & $12 a_{0333}$ & $12 a_{0484}$ & $12 a_{0688}$ \\
\hline $11 a_{18}$ & $11 a_{172}$ & $11 n_{6}$ & $11 n_{166}$ & $12 a_{0074}$ & $12 a_{0164}$ & $12 a_{0334}$ & $12 a_{0489}$ & $12 a_{0692}$ \\
\hline $11 a_{19}$ & $11 a_{173}$ & $11 n_{7}$ & $11 n_{182}$ & $12 a_{0075}$ & $12 a_{0166}$ & $12 a_{0336}$ & $12 a_{0490}$ & $12 a_{0693}$ \\
\hline $11 a_{20}$ & $11 a_{231}$ & $11 n_{8}$ & $12 a_{0001}$ & $12 a_{0076}$ & $12 a_{0167}$ & $12 a_{0337}$ & $12 a_{0491}$ & $12 a_{0694}$ \\
\hline $11 a_{22}$ & $11 a_{232}$ & $11 n_{9}$ & $12 a_{0002}$ & $12 a_{0079}$ & $12 a_{0177}$ & $12 a_{0341}$ & $12 a_{0493}$ & $12 a_{0697}$ \\
\hline $11 a_{24}$ & $11 a_{244}$ & $11 n_{10}$ & $12 a_{0004}$ & $12 a_{0080}$ & $12 a_{0182}$ & $12 a_{0343}$ & $12 a_{0494}$ & $12 a_{0698}$ \\
\hline $11 a_{25}$ & $11 a_{250}$ & $11 n_{11}$ & $12 a_{0005}$ & $12 a_{0088}$ & $12 a_{0184}$ & $12 a_{0344}$ & $12 a_{0495}$ & $12 a_{0699}$ \\
\hline
\end{tabular}




\begin{tabular}{|c|c|c|c|c|c|c|c|c|}
\hline $12 a_{0701}$ & $12 a_{0867}$ & $12 a_{0959}$ & $12 a_{1076}$ & $12 a_{1211}$ & $12 n_{0018}$ & $12 n_{0089}$ & $12 n_{0141}$ & $12 n_{0224}$ \\
\hline $12 a_{0702}$ & $12 a_{0868}$ & $12 a_{0960}$ & $12 a_{1078}$ & $12 a_{1212}$ & $12 n_{0019}$ & $12 n_{0090}$ & $12 n_{0156}$ & $12 n_{0225}$ \\
\hline $12 a_{0703}$ & $12 a_{0871}$ & $12 a_{0961}$ & $12 a_{1079}$ & $12 a_{1213}$ & $12 n_{0020}$ & $12 n_{0091}$ & $12 n_{0157}$ & $12 n_{0226}$ \\
\hline $12 a_{0705}$ & $12 a_{0872}$ & $12 a_{0964}$ & $12 a_{1080}$ & $12 a_{1215}$ & $12 n_{0021}$ & $12 n_{0092}$ & $12 n_{0158}$ & $12 n_{0227}$ \\
\hline $12 a_{0706}$ & $12 a_{0873}$ & $12 a_{0965}$ & $12 a_{1081}$ & $12 a_{1216}$ & $12 n_{0022}$ & $12 n_{0093}$ & $12 n_{0173}$ & $12 n_{0228}$ \\
\hline $12 a_{0707}$ & $12 a_{0874}$ & $12 a_{0966}$ & $12 a_{1083}$ & $12 a_{1217}$ & $12 n_{0023}$ & $12 n_{0094}$ & $12 n_{0174}$ & $12 n_{0229}$ \\
\hline $12 a_{0708}$ & $12 a_{0875}$ & $12 a_{0970}$ & $12 a_{1087}$ & $12 a_{1218}$ & $12 n_{0024}$ & $12 n_{0095}$ & $12 n_{0175}$ & $12 n_{0230}$ \\
\hline $12 a_{0709}$ & $12 a_{0884}$ & $12 a_{0971}$ & $12 a_{1088}$ & $12 a_{1219}$ & $12 n_{0026}$ & $12 n_{0096}$ & $12 n_{0176}$ & $12 n_{0231}$ \\
\hline $12 a_{0710}$ & $12 a_{0885}$ & $12 a_{0975}$ & $12 a_{1091}$ & $12 a_{1220}$ & $12 n_{0027}$ & $12 n_{0097}$ & $12 n_{0177}$ & $12 n_{0232}$ \\
\hline $12 a_{0741}$ & $12 a_{0886}$ & $12 a_{0976}$ & $12 a_{1092}$ & $12 a_{1221}$ & $12 n_{0028}$ & $12 n_{0098}$ & $12 n_{0178}$ & $12 n_{0245}$ \\
\hline $12 a_{0750}$ & $12 a_{0888}$ & $12 a_{0979}$ & $12 a_{1093}$ & $12 a_{1222}$ & $12 n_{0029}$ & $12 n_{0099}$ & $12 n_{0179}$ & $12 n_{0246}$ \\
\hline $12 a_{0755}$ & $12 a_{0891}$ & $12 a_{0981}$ & $12 a_{1095}$ & $12 a_{1225}$ & $12 n_{0030}$ & $12 n_{0100}$ & $12 n_{0180}$ & $12 n_{0247}$ \\
\hline $12 a_{0771}$ & $12 a_{0893}$ & $12 a_{0982}$ & $12 a_{1096}$ & $12 a_{1229}$ & $12 n_{0031}$ & $12 n_{0101}$ & $12 n_{0181}$ & $12 n_{0252}$ \\
\hline $12 a_{0798}$ & $12 a_{0894}$ & $12 a_{0986}$ & $12 a_{1097}$ & $12 a_{1230}$ & $12 n_{0032}$ & $12 n_{0102}$ & $12 n_{0182}$ & $12 n_{0253}$ \\
\hline $12 a_{0801}$ & $12 a_{0895}$ & $12 a_{0987}$ & $12 a_{1098}$ & $12 a_{1231}$ & $12 n_{0033}$ & $12 n_{0103}$ & $12 n_{0183}$ & $12 n_{0254}$ \\
\hline $12 a_{0804}$ & $12 a_{0898}$ & $12 a_{0990}$ & $12 a_{1099}$ & $12 a_{1232}$ & $12 n_{0034}$ & $12 n_{0104}$ & $12 n_{0184}$ & $12 n_{0255}$ \\
\hline $12 a_{0811}$ & $12 a_{0899}$ & $12 a_{0994}$ & $12 a_{1100}$ & $12 a_{1237}$ & $12 n_{0049}$ & $12 n_{0105}$ & $12 n_{0185}$ & $12 n_{0256}$ \\
\hline $12 a_{0812}$ & $12 a_{0900}$ & $12 a_{0999}$ & $12 a_{1101}$ & $12 a_{1246}$ & $12 n_{0050}$ & $12 n_{0106}$ & $12 n_{0186}$ & $12 n_{0257}$ \\
\hline $12 a_{0813}$ & $12 a_{0903}$ & $12 a_{1000}$ & $12 a_{1102}$ & $12 a_{1248}$ & $12 n_{0051}$ & $12 n_{0107}$ & $12 n_{0187}$ & $12 n_{0258}$ \\
\hline $12 a_{0814}$ & $12 a_{0906}$ & $12 a_{1002}$ & $12 a_{1103}$ & $12 a_{1249}$ & $12 n_{0052}$ & $12 n_{0108}$ & $12 n_{0188}$ & $12 n_{0259}$ \\
\hline $12 a_{0815}$ & $12 a_{0909}$ & $12 a_{1003}$ & $12 a_{1105}$ & $12 a_{1251}$ & $12 n_{0053}$ & $12 n_{0109}$ & $12 n_{0189}$ & $12 n_{0260}$ \\
\hline $12 a_{0816}$ & $12 a_{0910}$ & $12 a_{1004}$ & $12 a_{1109}$ & $12 a_{1252}$ & $12 n_{0055}$ & $12 n_{0110}$ & $12 n_{0190}$ & $12 n_{0261}$ \\
\hline $12 a_{0817}$ & $12 a_{0911}$ & $12 a_{1007}$ & $12 a_{1110}$ & $12 a_{1253}$ & $12 n_{0056}$ & $12 n_{0111}$ & $12 n_{0191}$ & $12 n_{0262}$ \\
\hline $12 a_{0818}$ & $12 a_{0912}$ & $12 a_{1008}$ & $12 a_{1111}$ & $12 a_{1254}$ & $12 n_{0057}$ & $12 n_{0112}$ & $12 n_{0192}$ & $12 n_{0263}$ \\
\hline $12 a_{0824}$ & $12 a_{0913}$ & $12 a_{1009}$ & $12 a_{1117}$ & $12 a_{1255}$ & $12 n_{0058}$ & $12 n_{0113}$ & $12 n_{0193}$ & $12 n_{0264}$ \\
\hline $12 a_{0825}$ & $12 a_{0914}$ & $12 a_{1010}$ & $12 a_{1119}$ & $12 a_{1256}$ & $12 n_{0059}$ & $12 n_{0114}$ & $12 n_{0194}$ & $2 n_{0265}$ \\
\hline $12 a_{0828}$ & $12 a_{0916}$ & $12 a_{1011}$ & $12 a_{1120}$ & $12 a_{1257}$ & $12 n_{0060}$ & $12 n_{0115}$ & $12 n_{0195}$ & $12 n_{0266}$ \\
\hline $12 a_{0829}$ & $12 a_{0917}$ & $12 a_{1012}$ & $12 a_{1121}$ & $12 a_{1260}$ & $12 n_{0061}$ & $12 n_{0116}$ & $12 n_{0196}$ & $12 n_{0267}$ \\
\hline $12 a_{0830}$ & $12 a_{0923}$ & $12 a_{1013}$ & $12 a_{1122}$ & $12 a_{1261}$ & $12 n_{0062}$ & $12 n_{0117}$ & $12 n_{0197}$ & $12 n_{0268}$ \\
\hline $12 a_{0831}$ & $12 a_{0924}$ & $12 a_{1014}$ & $12 a_{1123}$ & $12 a_{1263}$ & $12 n_{0063}$ & $12 n_{0118}$ & $12 n_{0201}$ & $12 n_{0269}$ \\
\hline $12 a_{0832}$ & $12 a_{0930}$ & $12 a_{1015}$ & $12 a_{1124}$ & $12 a_{1266}$ & $12 n_{0064}$ & $12 n_{0119}$ & $12 n_{0202}$ & $12 n_{0270}$ \\
\hline $12 a_{0833}$ & $12 a_{0931}$ & $12 a_{1016}$ & $12 a_{1156}$ & $12 a_{1267}$ & $12 n_{0066}$ & $12 n_{0120}$ & $12 n_{0203}$ & $12 n_{0290}$ \\
\hline $12 a_{0834}$ & $12 a_{0933}$ & $12 a_{1017}$ & $12 a_{1167}$ & $12 a_{1269}$ & $12 n_{0067}$ & $12 n_{0122}$ & $12 n_{0204}$ & $12 n_{0291}$ \\
\hline $12 a_{0841}$ & $12 a_{0934}$ & $12 a_{1018}$ & $12 a_{1173}$ & $12 a_{1270}$ & $12 n_{0068}$ & $12 n_{0123}$ & $12 n_{0205}$ & $12 n_{0292}$ \\
\hline $12 a_{0844}$ & $12 a_{0936}$ & $12 a_{1019}$ & $12 a_{1175}$ & $12 a_{1271}$ & $12 n_{0069}$ & $12 n_{0124}$ & $12 n_{0206}$ & $12 n_{0315}$ \\
\hline $12 a_{0845}$ & $12 a_{0939}$ & $12 a_{1020}$ & $12 a_{1181}$ & $12 a_{1272}$ & $12 n_{0070}$ & $12 n_{0125}$ & $12 n_{0207}$ & $12 n_{0317}$ \\
\hline $12 a_{0846}$ & $12 a_{0940}$ & $12 a_{1021}$ & $12 a_{1184}$ & $12 a_{1288}$ & $12 n_{0071}$ & $12 n_{0126}$ & $12 n_{0208}$ & $12 n_{0320}$ \\
\hline $12 a_{0847}$ & $12 a_{0941}$ & $12 a_{1036}$ & $12 a_{1185}$ & $12 n_{0001}$ & $12 n_{0072}$ & $12 n_{0127}$ & $12 n_{0209}$ & $12 n_{0326}$ \\
\hline $12 a_{0848}$ & $12 a_{0942}$ & $12 a_{1038}$ & $12 a_{1186}$ & $12 n_{0002}$ & $12 n_{0073}$ & $12 n_{0128}$ & $12 n_{0210}$ & $12 n_{0329}$ \\
\hline $12 a_{0849}$ & $12 a_{0944}$ & $12 a_{1043}$ & $12 a_{1187}$ & $12 n_{0003}$ & $12 n_{0074}$ & $12 n_{0129}$ & $12 n_{0211}$ & $12 n_{0330}$ \\
\hline $12 a_{0850}$ & $12 a_{0945}$ & $12 a_{1050}$ & $12 a_{1190}$ & $12 n_{0004}$ & $12 n_{0075}$ & $12 n_{0130}$ & $12 n_{0212}$ & $12 n_{0331}$ \\
\hline $12 a_{0851}$ & $12 a_{0946}$ & $12 a_{1053}$ & $12 a_{1191}$ & $12 n_{0005}$ & $12 n_{0076}$ & $12 n_{0131}$ & $12 n_{0213}$ & $12 n_{0335}$ \\
\hline $12 a_{0852}$ & $12 a_{0947}$ & $12 a_{1057}$ & $12 a_{1192}$ & $12 n_{0006}$ & $12 n_{0080}$ & $12 n_{0132}$ & $12 n_{0214}$ & $12 n_{0336}$ \\
\hline $12 a_{0853}$ & $12 a_{0948}$ & $12 a_{1059}$ & $12 a_{1193}$ & $12 n_{0007}$ & $12 n_{0081}$ & $12 n_{0133}$ & $12 n_{0215}$ & $12 n_{0344}$ \\
\hline $12 a_{0854}$ & $12 a_{0949}$ & $12 a_{1061}$ & $12 a_{1194}$ & $12 n_{0008}$ & $12 n_{0082}$ & $12 n_{0134}$ & $12 n_{0216}$ & $12 n_{0345}$ \\
\hline $12 a_{0859}$ & $12 a_{0951}$ & $12 a_{1062}$ & $12 a_{1196}$ & $12 n_{0009}$ & $12 n_{0083}$ & $12 n_{0135}$ & $12 n_{0217}$ & $12 n_{0346}$ \\
\hline $12 a_{0860}$ & $12 a_{0952}$ & $12 a_{1064}$ & $12 a_{1202}$ & $12 n_{0010}$ & $12 n_{0084}$ & $12 n_{0136}$ & $12 n_{0219}$ & $12 n_{0364}$ \\
\hline $12 a_{0861}$ & $12 a_{0953}$ & $12 a_{1065}$ & $12 a_{1203}$ & $12 n_{0014}$ & $12 n_{0085}$ & $12 n_{0137}$ & $12 n_{0220}$ & $12 n_{0365}$ \\
\hline $12 a_{0862}$ & $12 a_{0956}$ & $12 a_{1067}$ & $12 a_{1204}$ & $12 n_{0015}$ & $12 n_{0086}$ & $12 n_{0138}$ & $12 n_{0221}$ & $12 n_{0376}$ \\
\hline $12 a_{0863}$ & $12 a_{0957}$ & $12 a_{1070}$ & $12 a_{1205}$ & $12 n_{0016}$ & $12 n_{0087}$ & $12 n_{0139}$ & $12 n_{0222}$ & $12 n_{0421}$ \\
\hline $12 a_{0866}$ & $12 a_{0958}$ & $12 a_{1074}$ & $12 a_{1209}$ & $12 n_{0017}$ & $12 n_{0088}$ & $12 n_{0140}$ & $12 n_{0223}$ & $12 n_{0422}$ \\
\hline
\end{tabular}


COMPUTING CLOSED ESSENTIAL SURFACES IN KNOT COMPLEMENTS

$\begin{array}{lllllllll}12 n_{0423} & 12 n_{0496} & 12 n_{0556} & 12 n_{0658} & 12 n_{0680} & 12 n_{0697} & 12 n_{0766} & 12 n_{0831} & 12 n_{0874} \\ 12 n_{0431} & 12 n_{0508} & 12 n_{0558} & 12 n_{0665} & 12 n_{0681} & 12 n_{0702} & 12 n_{0769} & 12 n_{0834} & 12 n_{0879} \\ 12 n_{0437} & 12 n_{0518} & 12 n_{0567} & 12 n_{0670} & 12 n_{0682} & 12 n_{0705} & 12 n_{0775} & 12 n_{0837} & 12 n_{0886} \\ 12 n_{0440} & 12 n_{0526} & 12 n_{0568} & 12 n_{0671} & 12 n_{0688} & 12 n_{0706} & 12 n_{0776} & 12 n_{0840} & 12 n_{0887} \\ 12 n_{0448} & 12 n_{0533} & 12 n_{0600} & 12 n_{0672} & 12 n_{0689} & 12 n_{0712} & 12 n_{0779} & 12 n_{0842} & 12 n_{0888} \\ 12 n_{0454} & 12 n_{0538} & 12 n_{0601} & 12 n_{0673} & 12 n_{0690} & 12 n_{0714} & 12 n_{0780} & 12 n_{0853} \\ 12 n_{0455} & 12 n_{0539} & 12 n_{0602} & 12 n_{0674} & 12 n_{0691} & 12 n_{0716} & 12 n_{0781} & 12 n_{0854} \\ 12 n_{0456} & 12 n_{0541} & 12 n_{0604} & 12 n_{0675} & 12 n_{0692} & 12 n_{0728} & 12 n_{0782} & 12 n_{0863} \\ 12 n_{0484} & 12 n_{0548} & 12 n_{0605} & 12 n_{0676} & 12 n_{0693} & 12 n_{0747} & 12 n_{0790} & 12 n_{0864} \\ 12 n_{0485} & 12 n_{0553} & 12 n_{0622} & 12 n_{0677} & 12 n_{0694} & 12 n_{0748} & 12 n_{0800} & 12 n_{0866} \\ 12 n_{0494} & 12 n_{0554} & 12 n_{0633} & 12 n_{0678} & 12 n_{0695} & 12 n_{0751} & 12 n_{0802} & 12 n_{0868} \\ 12 n_{0495} & 12 n_{0555} & 12 n_{0642} & 12 n_{0679} & 12 n_{0696} & 12 n_{0753} & 12 n_{0826} & 12 n_{0869}\end{array}$

School of Mathematics and Physics, The University of Queensland, Brisbane QLD 4072, Australia

E-mail address: bab@maths.uq.edu.au

School of Mathematics and Statistics, The University of Sydney, Sydney NSW 2006, Australia

E-mail address: coward@maths.usyd.edu.au

School of Mathematics and Statistics, The University of Sydney, Sydney NSW 2006, Australia

E-mail address: tillmann@maths.usyd.edu.au 